2013

\title{
Overview of physics results from MAST towards ITER/DEMO and the MAST Upgrade
}

H. Meyer

R. J. Akers

S. Y. Allan

S. Mordijck

William and Mary

Follow this and additional works at: https://scholarworks.wm.edu/aspubs

\section{Recommended Citation}

Meyer, H., Abel, I. G., Akers, R. J., Allan, A., Allan, S. Y., Appel, L. C., ... \& Bradley, J. W. (2013). Overview of physics results from MAST towards ITER/DEMO and the MAST Upgrade. Nuclear Fusion, 53(10), 104008.

This Article is brought to you for free and open access by the Arts and Sciences at W\&M ScholarWorks. It has been accepted for inclusion in Arts \& Sciences Articles by an authorized administrator of W\&M ScholarWorks. For more information, please contact scholarworks@wm.edu. 


\section{Overview of physics results from MAST towards ITER/DEMO and the MAST Upgrade}

H. Meyer ${ }^{1}$, I.G. Abel ${ }^{2}$, R.J. Akers ${ }^{1}$, A. Allan ${ }^{3}$, S.Y. Allan ${ }^{1}$, L.C. Appel ${ }^{1}$, O. Asunta ${ }^{4}$, M. Barnes ${ }^{2,5}$, N.C. Barratt ${ }^{3}$, N. Ben Ayed ${ }^{1}$, J.W. Bradley ${ }^{6}$, J. Canik $^{7}$, P. Cahyna ${ }^{8}$, M. Cecconello ${ }^{9}$, C.D. Challis ${ }^{1}$, I.T. Chapman ${ }^{1}$, D. Ciric ${ }^{1}$, G. Colyer ${ }^{1}$, N.J. Conway ${ }^{1}$, M. Cox ${ }^{1}$, B.J. Crowley ${ }^{1}$, S.C. Cowley ${ }^{1}$, G. Cunningham ${ }^{1}$, A. Danilov ${ }^{10}$, A. Darke ${ }^{1}$, M.F.M. De Bock ${ }^{11}$, G. De Temmerman ${ }^{12}$, R.O. Dendy ${ }^{1}$, P. Denner ${ }^{3}$, D. Dickinson ${ }^{1}$, A.Y. Dnestrovsky ${ }^{10}$, Y. Dnestrovsky ${ }^{10}$, M.D. Driscoll ${ }^{1}$, B. Dudson ${ }^{3}$, D. Dunai ${ }^{13}$, M. Dunstan ${ }^{1}$, P. Dura ${ }^{14}$, S. Elmore ${ }^{1,6}$, A.R. Field ${ }^{1}$, G. Fishpool ${ }^{1}$, S. Freethy ${ }^{1}$, W. Fundamenski ${ }^{1}$, L. Garzotti ${ }^{1}$, Y.C. Ghim ${ }^{1,2}$, K.J. Gibson ${ }^{3}$, M.P. Gryaznevich ${ }^{1}$, J. Harrison ${ }^{1}$, E. Havlíčková ${ }^{1}$, N.C. Hawkes ${ }^{1}$, W.W. Heidbrink ${ }^{15}$, T.C. Hender ${ }^{1}$, E. Highcock ${ }^{2}$, D. Higgins ${ }^{14}$, P. Hill ${ }^{2}$, B. Hnat ${ }^{14}$, M.J. Hole ${ }^{16}$, J. Horáček ${ }^{8}$, D.F. Howell ${ }^{1}$, K. Imada ${ }^{3}$, O. Jones ${ }^{17}$, E. Kaveeva ${ }^{18}$, D. Keeling ${ }^{1}$, A. Kirk ${ }^{1}$, M. Kočan ${ }^{19}$, R.J. Lake ${ }^{14}$, M. Lehnen ${ }^{20}$, H.J. Leggate ${ }^{21}$, Y. Liang ${ }^{20}$, M.K. Lilley ${ }^{22}$, S.W. Lisgo ${ }^{23}$, Y.Q. Liu ${ }^{1}$, B. Lloyd ${ }^{1}$, G.P. Maddison ${ }^{1}$, J. Mailloux ${ }^{1}$, R. Martin ${ }^{1}$, G.J. McArdle ${ }^{1}$, K.G. McClements ${ }^{1}$, B. McMillan ${ }^{14}$, C. Michael $^{1}$, F. Militello ${ }^{1}$, P. Molchanov ${ }^{18}$, S. Mordijck ${ }^{24}$, T. Morgan ${ }^{12}$, A.W. Morris ${ }^{1}$, D.G. Muir ${ }^{1}$, E. Nardon ${ }^{25}$, V. Naulin ${ }^{26}$, G. Naylor ${ }^{1}$, A.H. Nielsen ${ }^{26}$, M.R. O'Brien ${ }^{1}$, T. O'Gorman ${ }^{3}$, S. Pamela ${ }^{27}$, F.I. Parra ${ }^{2,5}$, A. Patel ${ }^{1}$, S.D. Pinches ${ }^{1,23}$, M.N. Price ${ }^{1}$, C.M. Roach ${ }^{1}$, J.R. Robinson ${ }^{14}$, M. Romanelli ${ }^{1}$, V. Rozhansky ${ }^{18}$, S. Saarelma ${ }^{1}$, S. Sangaroon ${ }^{8}$, A. Saveliev ${ }^{28}$, R. Scannell ${ }^{1}$, J. Seidl ${ }^{7}$, S.E. Sharapov ${ }^{1}$, A.A. Schekochihin ${ }^{2}$, V. Shevchenko ${ }^{1}$, S. Shibaev ${ }^{1}$, D. Stork ${ }^{1}$, J. Storrs ${ }^{1}$, A. Sykes ${ }^{1}$, G.J. Tallents ${ }^{3}$, P. Tamain ${ }^{25}$, D. Taylor ${ }^{1}$, D. Temple ${ }^{22}$, N. Thomas-Davies ${ }^{1}$, A. Thornton ${ }^{1}$, M.R. Turnyanskiy ${ }^{1}$, M. Valovič ${ }^{1}$, R.G.L. Vann ${ }^{3}$, E. Verwichte ${ }^{14}$, P. Voskoboynikov ${ }^{18}$, G. Voss ${ }^{1}$, S.E.V. Warder ${ }^{1}$, H.R. Wilson ${ }^{3}$, I. Wodniak $^{8}$, S. Zoletnik ${ }^{13}$, R. Zagôrski ${ }^{29}$ and the MAST and NBI Teams

\footnotetext{
${ }^{1}$ EURATOM/CCFE Fusion Association, Culham Science Centre, Abingdon, UK

${ }^{2}$ Rudolf Peierls Centre for Theoretical Physics, University of Oxford, Oxford, UK

${ }^{3}$ Department of Physics, University of York, Heslington, York, UK

${ }^{4}$ Aalto University, Association EURATOM-TEKES, Espoo, Finland

${ }^{5}$ MIT Plasma Science and Fusion Center, Cambridge, MA 02139, USA

${ }^{6}$ Department of Electrical Engineering and Electronics, University of Liverpool, Brownlow Hill, Liverpool, UK

${ }^{7}$ Oak Ridge National Laboratory, Oak Ridge, TN, USA

${ }^{8}$ Institute of Plasma Physics AS CR vvi, Association EURATOM/IPP.CR, Prague, Czech

Republic

${ }^{9}$ EURATOM-VR Association, Uppsala University, SE-75120 Uppsala, Sweden

${ }^{10}$ Russian Research Centre, Kruchatov Institute, Institute of Nuclear Fusion, Moscow, Russia

${ }^{11}$ Department of Applied Physics, Eindhoven University of Technology, Eindhoven,

The Netherlands

${ }^{12}$ DIFFER, Association EURATOM-DIFFER, Nieuwegein, The Netherlands
} 
${ }^{13}$ KFKI-RMKI, Association EURATOM, Pf. 49, H-1525 Budapest, Hungary

${ }^{14}$ Centre for Fusion, Space and Astrophysics, Department of Physics, Warwick University,

Coventry, UK

${ }^{15}$ School of Physical Sciences, University of California, Irvine, CA 92697, USA

${ }^{16}$ Plasma Research Laboratory, Research School of Physical Science and Engineering,

Australian National University, Canberra, ACT 0200, Australia

${ }^{17}$ Department of Physics, University of Durham, Durham DH1 3LE, UK

${ }^{18}$ Department of Plasma Physics, St Petersburg State Polytechnical University, St Petersburg, Russia

${ }^{19}$ Max-Planck-Institut für Plasmaphysik, EURATOM Association, Garching, Germany

${ }^{20}$ Association EURATOM-FZ Jülich, Trilateral Euregio Cluster, D-52425 Jülich, Germany

${ }^{21}$ EURATOM/DCU Fusion Association, Dublin City University, Glasnevin, Dublin, Ireland

${ }^{22}$ Imperial College of Science, Technology and Medicine, London, UK

${ }^{23}$ ITER Organization, CS 90 046, 13067 St Paul lez Durance Cedex, France

${ }^{24}$ The College of William and Mary, McGlothlin-Street Hall, Williamsburg, VA 23187, USA

${ }^{25}$ CEA-Cadarache, Association Euratom-CEA, 13108 St Paul-lez-Durance, France

${ }^{26}$ Association EURATOM/Ris $\emptyset$, National Laboratory for Sustainable Energy, OPL-128, PO

Box 49, DK-4000 Roskilde, Denmark

${ }^{27}$ IIFS-PIIM Aix Marseille Université CNRS, 13397 Marseille Cedex20, France

${ }^{28}$ A.F. Ioffe Physico-Technical Institute, St Petersburg, Russia

${ }^{29}$ Association EURATOM/IPPLM, Institute of Plasma Physics and Laser Microfusion,

Warsaw, Poland

E-mail: Hendrik.Meyer@ccfe.ac.uk

Received 18 December 2012, accepted for publication 20 August 2013

Published 26 September 2013

Online at stacks.iop.org/NF/53/104008

\begin{abstract}
New diagnostic, modelling and plant capability on the Mega Ampère Spherical Tokamak (MAST) have delivered important results in key areas for ITER/DEMO and the upcoming MAST Upgrade, a step towards future ST devices on the path to fusion currently under procurement. Micro-stability analysis of the pedestal highlights the potential roles of micro-tearing modes and kinetic ballooning modes for the pedestal formation. Mitigation of edge localized modes (ELM) using resonant magnetic perturbation has been demonstrated for toroidal mode numbers $n=3,4,6$ with an ELM frequency increase by up to a factor of 9, compatible with pellet fuelling. The peak heat flux of mitigated and natural ELMs follows the same linear trend with ELM energy loss and the first ELM-resolved $T_{\mathrm{i}}$ measurements in the divertor region are shown. Measurements of flow shear and turbulence dynamics during L-H transitions show filaments erupting from the plasma edge whilst the full flow shear is still present. Off-axis neutral beam injection helps to strongly reduce the redistribution of fast-ions due to fishbone modes when compared to on-axis injection. Low- $k$ ion-scale turbulence has been measured in L-mode and compared to global gyro-kinetic simulations. A statistical analysis of principal turbulence time scales shows them to be of comparable magnitude and reasonably correlated with turbulence decorrelation time. $T_{\mathrm{e}}$ inside the island of a neoclassical tearing mode allow the analysis of the island evolution without assuming specific models for the heat flux. Other results include the discrepancy of the current profile evolution during the current ramp-up with solutions of the poloidal field diffusion equation, studies of the anomalous Doppler resonance compressional Alfvén eigenmodes, disruption mitigation studies and modelling of the new divertor design for MAST Upgrade. The novel 3D electron Bernstein synthetic imaging shows promising first data sensitive to the edge current profile and flows.
\end{abstract}

\section{Introduction}

Future magnetic confinement fusion devices face major challenges in the areas of plasma exhaust, pedestal and edge localized mode (ELM) physics as well as fast particle and current drive physics. MAST [1] $\left(A=\varepsilon^{-1}=R / a=\right.$ $\left.0.85 \mathrm{~m} / 0.65 \mathrm{~m} \sim 1.3, I_{\mathrm{p}} \leqslant 1.5 \mathrm{MA}\right)$, one of the two leading spherical tokamaks (STs) in the world (similar to NSTX [2]), is well suited to advance the physics basis for ITER ${ }^{30}$ [3], DEMO or an ST based component test facility (CTF) [4-6] in these areas $[7,8]$. The MAST Upgrade project $[9,10]$ (under procurement) will strengthen this even further with the new

${ }^{30}$ www.iter.org. upper and lower divertor, the off-axis neural beam heating, the new centre column allowing for a $90 \%$ higher flux swing, a $50 \%$ higher toroidal field with five times higher $I^{2} t$ and higher shaping capabilities. These substantial upgrades to the load assembly, the heating system and the power supplies will enable detailed studies of novel divertor concepts including Super-X [11] and Snow flake [12], fast particle physics and current drive in a much wider operating space. On MAST the hot $T_{\mathrm{i}} \leqslant 3 \mathrm{keV}, T_{\mathrm{e}} \leqslant 2 \mathrm{keV}$, dense $n_{\mathrm{e}}=(0.1-1) \times 10^{20} \mathrm{~m}^{-3}$ and highly shaped $(\delta \leqslant 0.5,1.6 \leqslant \kappa \leqslant 2.5)$ plasmas are accessed at moderate toroidal field $B_{\mathrm{t}}(R=0.7 \mathrm{~m}) \leqslant 0.62 \mathrm{~T}$ giving access to regimes at high $\beta=2 \mu_{0} p / B^{2}$, strong rotation, where finite Larmor radius, $\rho_{\mathrm{i}}$, and magnetic field 

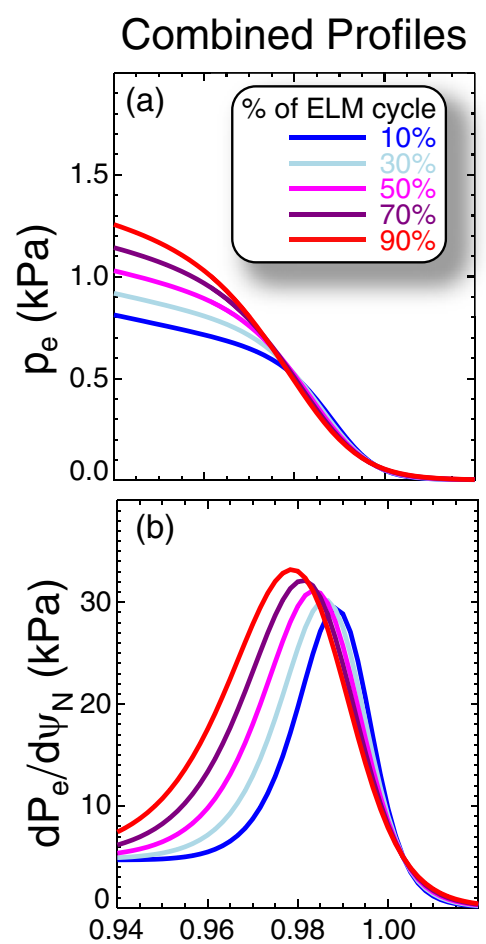

Individual Profiles
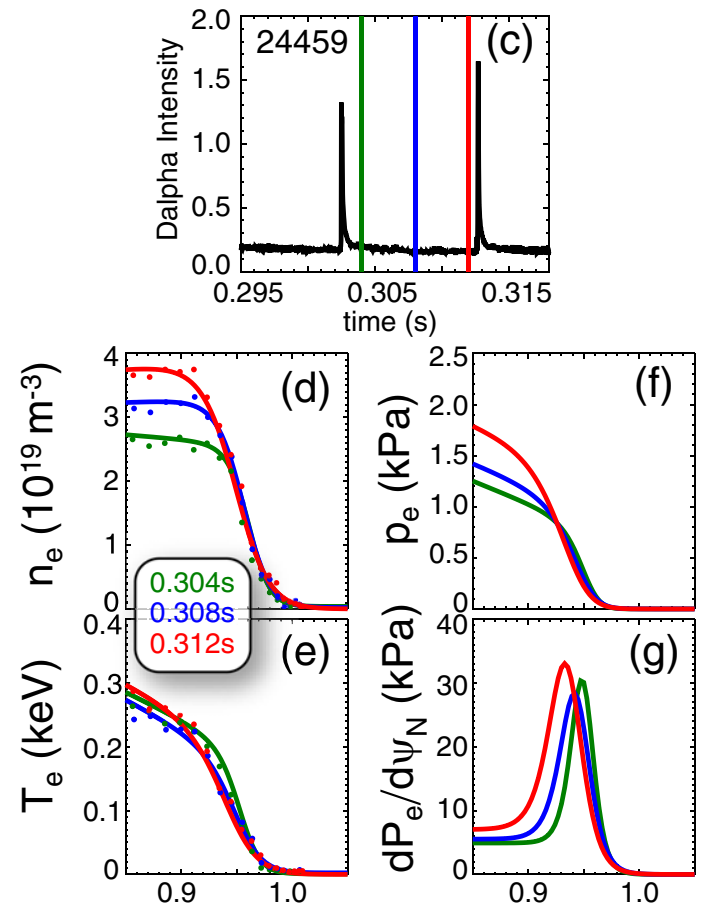

Normalised pol. flux

Figure 1. Profile evolution of the $(a)$ electron pressure and $(b)$ its gradient during a type-I ELM cycle constructed from 50 profiles in three similar shots as well as $(d)-(f)$ individual data with error bars and fits at three different times during one ELM cycle characterized by $(c)$ the $D_{\alpha}$ intensity.

curvature effects are enhanced. Apart from this ST unique parameter regime MAST plasmas also show many similarities to conventional aspect ratio tokamaks. Research during the last two years has delivered important results for the ITER physics basis with respect to ELM mitigation (section 5), fast particle physics (section 6), L-H thresholds (section 5), heat loads (section 5) and neoclassical tearing modes (NTMs) (section 8). Furthermore, MAST data also helped to resolve critical design issues for the Upgrade such as the thermal performance of the sliding joint design for the higher toroidal field and longer pulse length by validating the modelled performance against data from prototype joints, or testing a controllable high field side mid-plane gas valve (section 9). New radial field power supplies for the vertical stabilization control have been incorporated into the Upgrade scope after extensive testing of the old power supplies on MAST and analysing the feedback response to ELMs. The increased understanding of the fast-ion redistribution with off-axis NBI (see section 6) suggests that the initially proposed beam geometry could be improved by angling one of the new beam lines upwards. The engineering implications of this are currently under investigation.

\section{Pedestal and ELMs}

The physics determining the width of the edge transport barrier in H-mode and therefore the width and height of the pedestal is not yet well understood. In recent years a predictive model for both the height and width of the pedestal has been developed [13], where the pedestal gradient is limited by kinetic ballooning modes (KBM) and the growth of the pedestal is ultimately limited by the ideal peeling-ballooning stability limit. On MAST the evolution of the electron pedestal between type-I ELMs has been investigated in detail using the recently upgraded Thomson scattering system [14-16].

Figure 1 shows the profile of the electron pressure and its gradient at different times during a type-I ELM cycle on MAST. The data in figures $1(a)$ and $(b)$ are constructed by combining the fitted data from 50 individual profiles at different stages of the ELM cycle from three similar shots and using least-squares fits of the fitting parameters to construct the inter ELM profiles. In a single inter ELM period the MAST Thomson scattering system can measure eight profiles using the eight lasers triggered with respect to an ELM event. In the discharges analysed here the normal time resolution of $\Delta t=4.33 \mathrm{~ms}$ was used leading to an average of three profiles in an inter ELM cycle (see figures $1(c)-(e)$ ). Using the method described in [15] of sorting profiles of different ELM cycles in three shots with respect to their time before the next ELM, fitting each profile with a modified tanh fit and deriving an average evolution of the fitting parameters through the ELM cycle in principle an arbitrary resolution can be achieved of a smoothed ELM cycle. The reconstructed average evolution agrees well with the limited number of profiles from individual laser pulses during one ELM cycle $[15,16]$ as can be seen from figures $1(d)-(g)$ showing the measured profiles with error bars and fits $(d)-(e)$ as well as the pressure and its gradient $(f)-(g)$ calculated from the fits at three times during the ELM cycle $(c)$. It is the pedestal width that evolves the most, rather than the steepness of gradient. Also the region of steepest gradient moves further inwards. With 


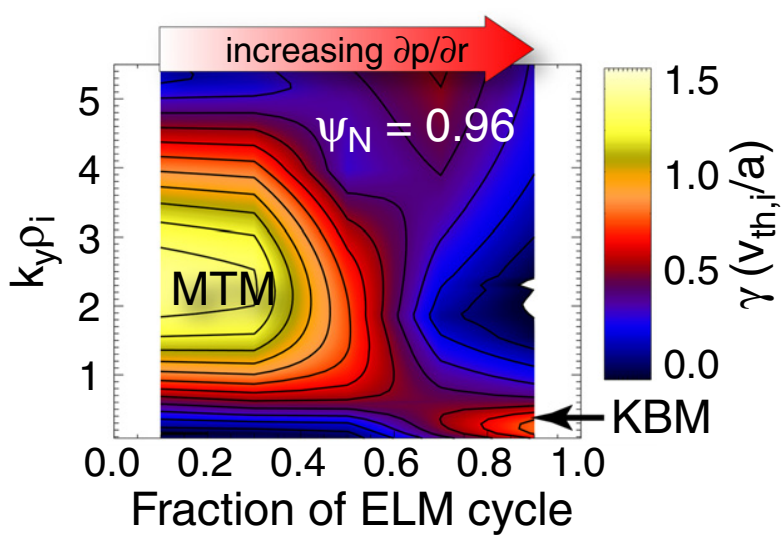

Figure 2. Normalized linear growth rate from GS2 as a function of time in the ELM cycle and normalized perpendicular wavelength $k_{y} \rho_{\mathrm{i}}\left(\rho_{\mathrm{i}}\right.$ : ion Larmor radius $)$.

respect to ideal peeling-ballooning stability this means that the widening of the pedestal region results in a decrease of the unstable pressure gradient limit [15]. A comparison between the pedestal evolution at low and high collisionality of the pedestal $v_{\star}^{\text {ped }}$ has been done, although in a non-dimensionless way. $v_{\star}=v_{\mathrm{ei}} \sqrt{m_{\mathrm{i}} /\left(k_{\mathrm{B}} T_{\mathrm{i}}\right)} \epsilon^{-3 / 2} q R$ is the electron ion collision frequency $v_{\mathrm{ei}}$ normalized to the ion bounce time $(\epsilon=a / R$ : inverse aspect ratio, $q=r B_{\mathrm{t}} / R B_{\theta}$ safety factor). Whilst the evolution of the pedestal pressure in time is similar at high and low $v_{\star}^{\text {ped }}$ the pedestal width and position evolve more slowly at low $v_{\star}^{\text {ped }}$. At high $v_{\star}^{\text {ped }}$ only $n_{\mathrm{e}}^{\text {ped }}$ increases at constant $T_{\mathrm{e}}^{\text {ped }}$.

A linear micro-stability analysis (at normalized perpendicular wavelength $\left.k_{y} \rho_{\mathrm{i}} \sim \mathcal{O}(1)\right)$ of the high $v_{\star}^{\text {ped }}$ profiles using the local gyro-kinetic (GK) code GS2 [17] shows that microtearing modes (MTMs) dominate the flatter gradient plateau region just inboard of the pedestal, and that KBMs dominate at the knee and in the steep gradient region of the pressure profile [15-18]. As can be seen from figure 2, a mode transition arises during the ELM cycle as the pressure pedestal broadens and the knee approaches $\psi_{\mathrm{N}}=\sqrt{\left(\psi-\psi_{0}\right) /\left(\psi_{a}-\psi_{0}\right)} \approx 0.96$ $\left(\psi_{0}\right.$ : poloidal flux at magnetic axis, $\psi_{a}$ : magnetic flux at last closed flux surface). The increasing $\partial n / \partial r$ and $\partial p / \partial r$ stabilize the MTMs until KBMs are driven unstable, which may be important in explaining the inward advance of the pedestal. In the steepest $\partial p / \partial r$ region KBMs become more stable due to increasing bootstrap current [16] and are fully stable at low $\nu_{\star}^{\text {ped }}[18,19]$. Studies using measured edge current profiles and GK modelling with global codes are needed to elucidate the role of the KBMs for the pedestal further.

\section{ELM mitigation}

In ITER and DEMO type-I ELMs need to be mitigated or suppressed to protect the plasma facing components [20], e.g. by using resonant magnetic perturbations (RMPs) [21-24]. The internal coil set on MAST has recently been upgraded from 12 coils to 18 internal coils, 12 below and 6 above the mid-plane, to study ELM mitigation with $n=2,3,4,6$ RMP fields.

In figure 3 an example of mitigation of type-I ELMs using an $n=6$ RMP is shown. Clearly with the application of

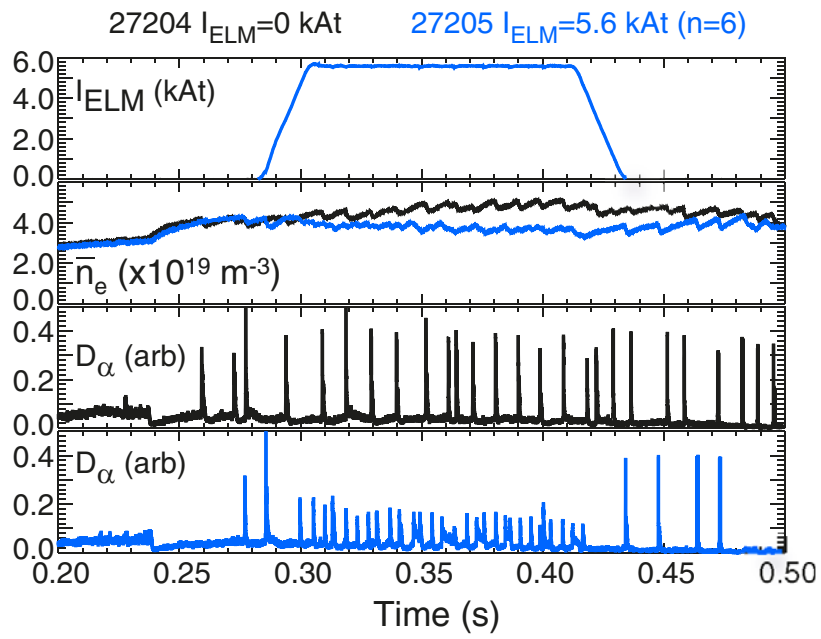

Figure 3. Example time traces for ELM mitigation (blue) with an $n=6$ perturbation. Shown are (a) coil current, $(b)$ line integrated density, (c) $\mathrm{D}_{\alpha}$ for unmitigated ELMs and $(d) \mathrm{D}_{\alpha}$ for mitigated ELMs.

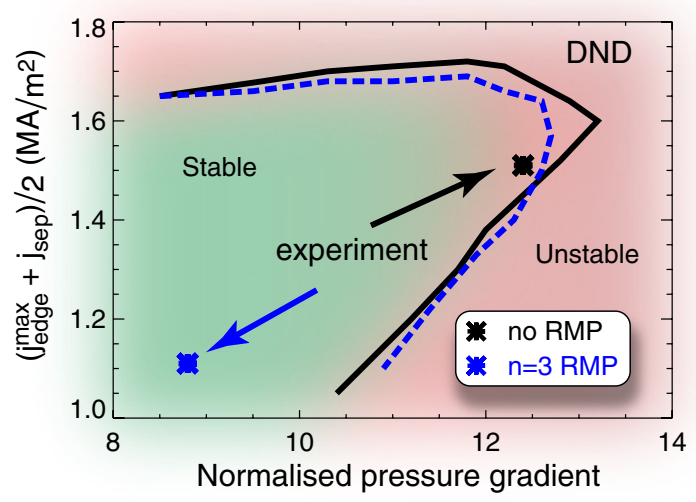

Figure 4. Stability diagram for similar discharges (black) without and (blue, dashed) with $n=3$ RMPs.

RMPs (blue) an increase of the ELM frequency $f_{\mathrm{ELM}}^{\mathrm{RMP}}$ over the natural type-I ELM frequency $f_{\mathrm{ELM}}^{\text {nat. }}$ is observed, here a factor $f_{\mathrm{ELM}}^{\mathrm{RMP}} / f_{\mathrm{ELM}}^{\text {nat. }} \approx 3$. When the perturbation is switched off $f_{\mathrm{ELM}}^{\text {nat. }}$ is recovered. Various scans with different mode spectra and plasma shape have shown that on MAST above a certain threshold perturbation $0.7 \times 10^{-4}<b_{\text {res }}^{r}<1.5 \times 10^{-4}$ the ELM frequency increases linearly with perturbation strength. Here, $b_{\text {res }}^{r}$ is the normalized radial component of the resonant perturbation field including the plasma response calculated with MARS-F [25]. The threshold itself depends on the applied spectrum. The ELM energy loss $\Delta W_{\mathrm{ELM}}$ is consistent with $f_{\mathrm{ELM}}^{\mathrm{RM}} \cdot \Delta W_{\mathrm{ELM}}=$ const. as for unmitigated ELMs. An increase of $f_{\mathrm{ELM}}^{\mathrm{RMP}} / f_{\mathrm{ELM}}^{\text {nat. }}$ up to 9 has been observed with a particular $n=3$ configuration. The upgraded coil set has now also the unique capability to perform a pitch angle scan of the $n=3$ perturbation at a fixed $q_{95}$ to test the resonance condition. This is done by keeping the current in the upper six coils constant, whilst changing the ratio of currents in the 12 lower coils operated in pairs. The sensitivity to the alignment together with modelling including the plasma response [26] suggests that the perturbation strength close to the X-point plays an 

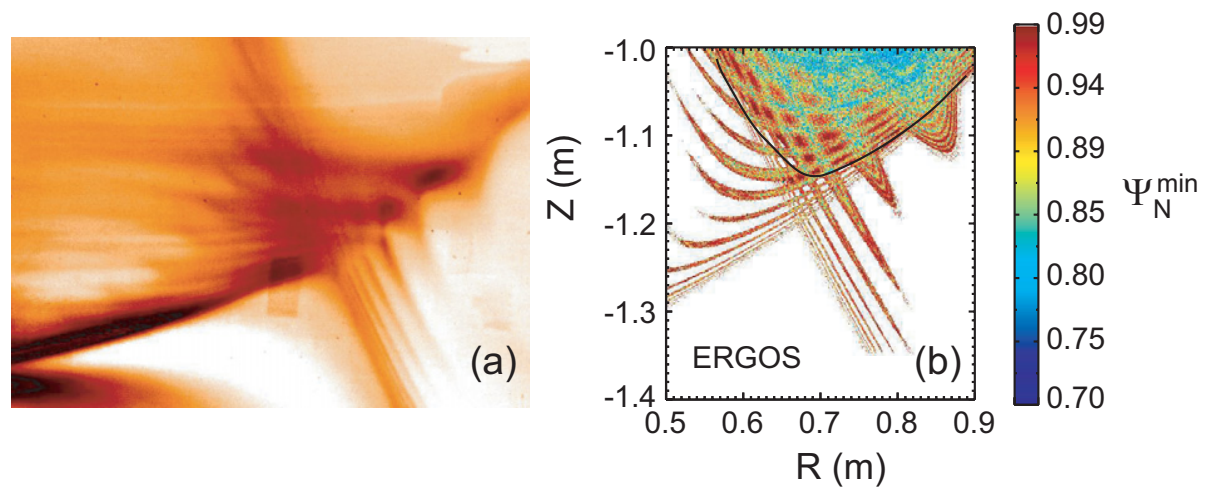

Figure 5. Lobe structures at the X-point $(a)$ observed with $n=6$ RMPs using narrow band visible imaging of $\mathrm{He}^{+}$emission and (b) modelled with ERGOS.

important role to affect the ELMs, although studies have as yet failed to identify a single parameter for ELM mitigation.

The application of the RMPs leads to a reduction in the edge pressure gradient [14]. Consequently, these profiles are well in the stable region of the peeling-ballooning stability diagram (see figure 4), despite the ELM frequency being higher. Hence, either the peeling-ballooning picture is no longer valid, or the usual 2D stability analysis is not applicable.

With perturbation fields above the threshold for ELM mitigation or density pump out (see above), lobe structures at the X-point appear as can be seen from figure 5(a) [27]. These structures are also predicted by vacuum field calculations of the perturbed equilibrium using the ERGOS code [28] (see figure $5(b)$ ). The application of RMPs also leads to periodic corrugation of the axisymmetric equilibrium. The capability on MAST to rotate the phase of the perturbation was used to compare the experimental profiles, i.e. from Thomson scattering and visible imaging, with a 3D equilibrium reconstruction using the ANIMEC code [29] showing good agreement between data and modelling. Including 3D effects into the stability analysis is likely to change the peelingballooning stability boundary. Addition of structures close to the $\mathrm{X}$-point in a 2D boundary for example reduces the pressure gradient at which peeling-ballooning stability is lost [30].

A study of high field side pellet fuelling with ITER-like deposition and pellet to ELM particle ratio during mitigated ELMs shows that the fuelling pellets trigger additional ELMs that could be larger than the mitigated ELMs [31]. In comparison to the ITER ideal fuelling model [32] examples with the same post-pellet particle loss rate as during the non-pellet phase as well as with five times larger post-pellet loss rate have been observed. The larger post-pellet loss rate is caused by the occurrence of an ELM that seems to consist of several ELM-like events after pellet injection. The reasons for the different pellet triggered ELMs under similar conditions is not fully understood and needs more experimental data on which to base hypotheses.

\section{4. $\mathrm{L}-\mathrm{H}$ transition}

A further critical issue for ITER is the access to H-mode. The dependence of the threshold power to access H-mode, $P_{\mathrm{L}-\mathrm{H}}$, on ELM mitigation techniques and on density is of particular interest. Previous results showed a detrimental effect with the application of $n=3$ RMPs on DIII-D, MAST and NSTX [33]. In these devices $P_{\mathrm{L}-\mathrm{H}}$ increased by up to a factor of two at perturbation strength needed for ELM mitigation. In contrast, the application of $n=4,6$ RMPs does not lead to a noticeable increase in $P_{\mathrm{L}-\mathrm{H}}$ on MAST and also mitigates the first ELM. Furthermore, shallow $(r / a>0.8)$ pellet injection is shown to be at least compatible with the L-H transition, if not beneficial [34]. The density dependence of the $\mathrm{L}-\mathrm{H}$ transition has been studied in plasmas without RMPs [14]. Power scans at three different densities, $\bar{n}_{\mathrm{e}}=(1.5 \pm 0.1) \times 10^{19} \mathrm{~m}^{-3}, \quad \bar{n}_{\mathrm{e}}=(2.0 \pm 0.1) \times 10^{19} \mathrm{~m}^{-3}$ and $\bar{n}_{\mathrm{e}}=(2.9 \pm 0.1) \times 10^{19} \mathrm{~m}^{-3}$, were performed by changing the level of NBI power on a shot to shot basis. Here, the power was only changed after the current ramp-up phase and the $\mathrm{L}-\mathrm{H}$ transition was suppressed until the current and density flat-top was reached, using the sensitivity of $P_{\mathrm{L}-\mathrm{H}}$ to the magnetic configuration $[35,36]$. This is to avoid effects of different current profile evolution on the relative $P_{\mathrm{L}-\mathrm{H}}$ measurements.

At the lowest density the available power of $P_{\mathrm{NBI}}=$ 3.5 MW was not sufficient to access a clear ELMy H-mode. Instead only an intermediate phase, $\tilde{H}$, is observed where the $\mathrm{D}_{\alpha}$ light dithers at frequencies of $4 \mathrm{kHz} \leqslant f_{\mathrm{D}_{\alpha}} \leqslant 5 \mathrm{kHz}$. This phase exists to very low density $\bar{n}_{\mathrm{e}} \approx 1.0 \times 10^{19} \mathrm{~m}^{-3}$ and over a wide range of input power. At the higher density ELMy H-mode is preceded by a similar phase, but the range in input power is reduced as well as the duration of this phase before the transition to ELMy H-mode occurs. This behaviour can be seen from figure 6 showing $(a)$ the $\mathrm{D}_{\alpha}$ traces at different densities and $(b)$ an indicative existence space in $P_{\text {loss }}$ and $\bar{n}_{\mathrm{e}}$. The periods $(\Delta t<200 \mu \mathrm{s})$ with low $\mathrm{D}_{\alpha}$ emission represent states of decreased edge turbulence measured with a reflectometer, beam emission spectroscopy (BES) and fast visible imaging. The periods are too short for the kinetic profiles to evolve significantly [37].

The dynamics of the toroidal and poloidal $\mathrm{He}^{+}$flow, representing the radial electric field, as the plasma enters the low turbulence periods in the $\tilde{H}$ phase is indistinguishable from the behaviour at the transition where the transport barrier can be sustained. Both the toroidal and poloidal velocity, measured by Doppler spectroscopy, indicate a more negative electric field with a stronger gradient in the edge region, just inside the last closed flux surface as can be seen from figures $7(a)$ and $(b)$. However, turbulent filaments are seen to erupt from the plasma $\sim 50 \mu$ s before the flow shear is lost. This would be consistent 


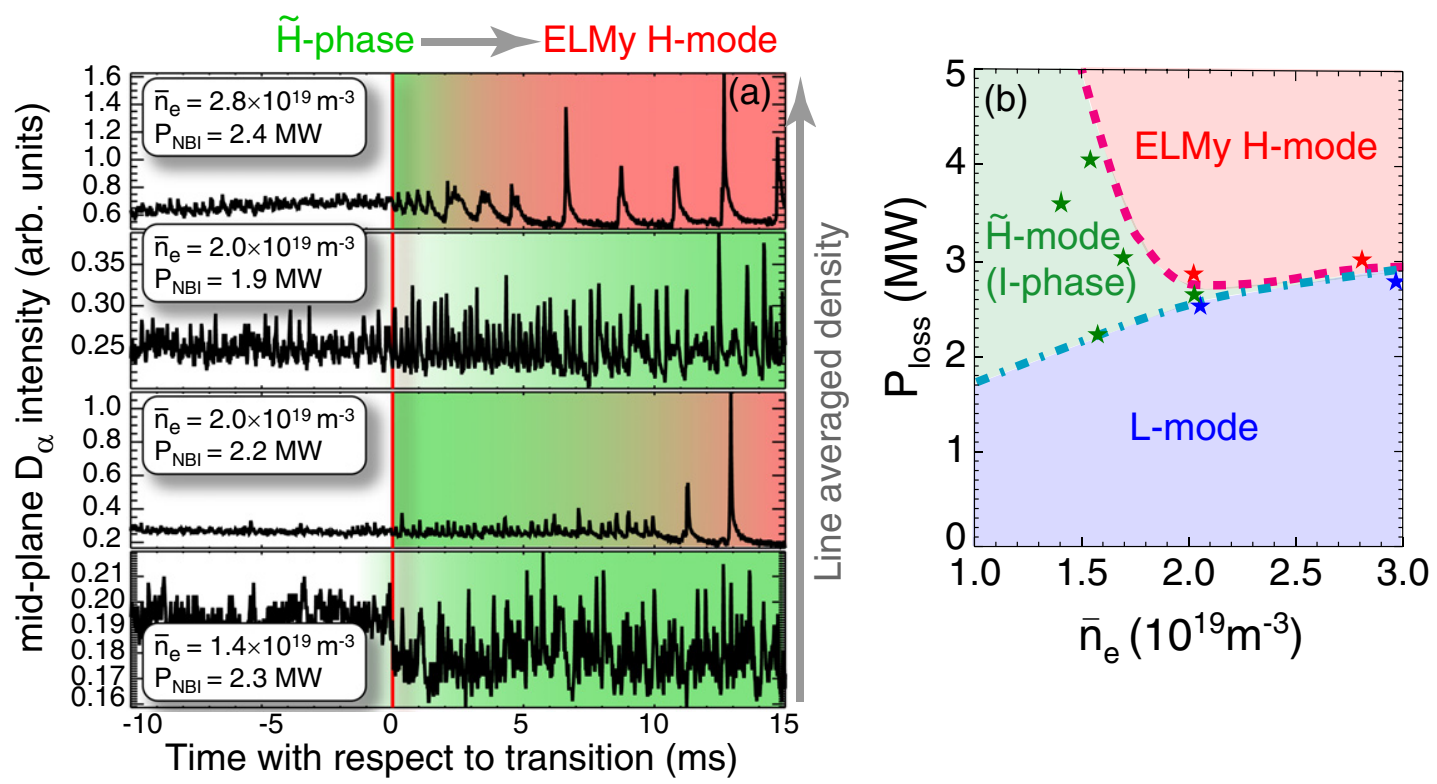

Figure 6. (a) $\mathrm{D}_{\alpha}$ traces for discharges at different densities close to the $\mathrm{L}-\mathrm{H}$ transition. The density increases from bottom to top. The $\tilde{H}$-phase is shown in green and the ELMy H-mode in red. The $\mathrm{L}$ to $\tilde{H}$-phase transition is marked by the vertical line. For the medium density case two power values are shown leading to a sustained $\tilde{H}$-phase with the lower power and a delayed transition to ELMy H-mode at higher power. (b) Indicative $P_{\text {loss }}$ versus $\bar{n}_{\mathrm{e}}$ existence space with measurement points overlaid.
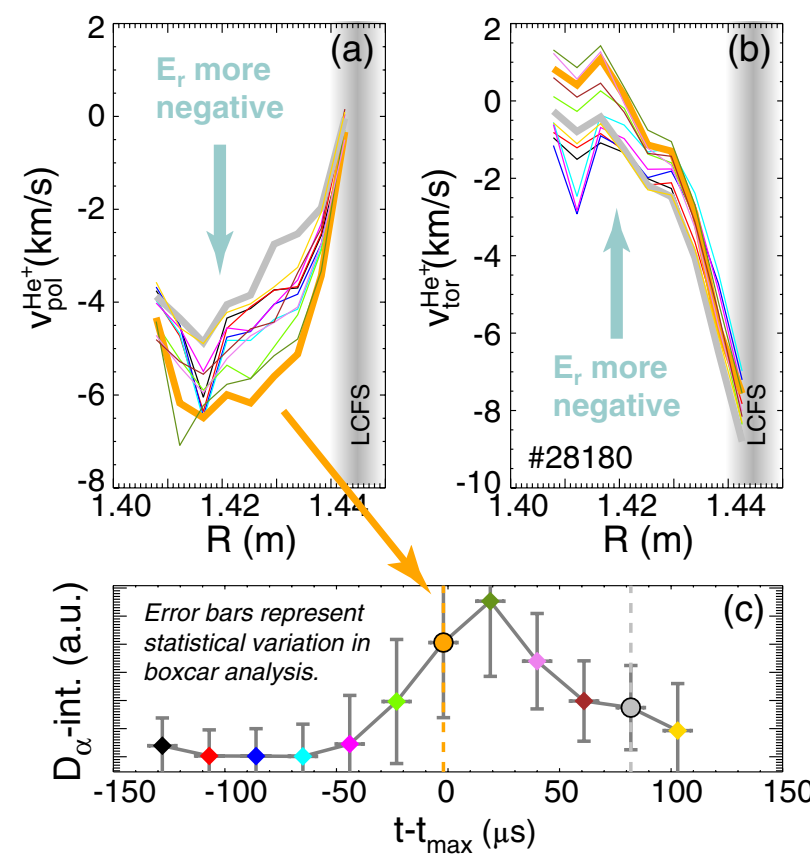

Figure 7. Boxcar averaged $(a)$ poloidal and $(b)$ toroidal $\mathrm{He}^{+}$flow profiles with respect to $(c)$ the boxcar averaged $\mathrm{D}_{\alpha}$ emission as proxy for the edge turbulence.

with a picture where the turbulence transports vorticity out of the edge region. Pending a detailed comparison this phase has some signatures of the I-phase observed in other devices $[38,39]$, a limit cycle oscillation between turbulence driven flow shear and plasma turbulence. The dynamics of the flow seems to be incompatible at least with simple predator-prey models [40,41], where the predator, turbulence driven flow shear, feeds on the prey, the turbulence. Here, a simple phase relation between flow shear and turbulence would be expected [42]. Geodesic acoustic modes (GAMs), also often connected to the I-phase [38], have been observed in $I_{\mathrm{p}}=0.4 \mathrm{MA}$ ohmic discharges on MAST and are found to be affected by RMPs suggesting a coupling to Alfvénic perturbations [43].

\section{Exhaust}

The conditions in the scrape-off-layer (SOL) set the boundary for the pedestal region, hence influencing both the $\mathrm{L}-\mathrm{H}$ transition [44] and the dynamics of the pedestal [45, 46]. All large future fusion devices face the challenge of how to deal with the high heat and particle fluxes impinging on the plasma facing components. In tokamaks and stellarators the SOL and divertor are key to this. The MAST Upgrade will incorporate, therefore, upper and lower divertors providing closure at the $\mathrm{X}$-points. A total of 16 coils allow for substantial changes of the magnetic geometry in the divertor volume [47, 48]. Here, the flux expansion at the target as well as further up-stream and the connection length can be changed. This also includes a so called Super-X configuration (SXD) [11]. The operation with a radially extended outer leg is in particular attractive for future high power ST devices, such as a CTF, although it could also mitigate the heat flux in conventional tokamaks. The tight aspect ratio leading to the smaller strike point radius in conventional divertor configurations leads also to a much stronger variation of $B_{\mathrm{t}}$ with $R$, which translates into a strong reduction of the parallel heat flux. The divertor design has been assessed with a variety of tools including edge modelling with SOLPS [49].

Selected results from an example simulation comparing SXD and a conventional configuration are shown in figure 8 [50]. In this simulation the flux expansion at the target leads to a strong reduction in $(b)$ peak energy flux with little change in 

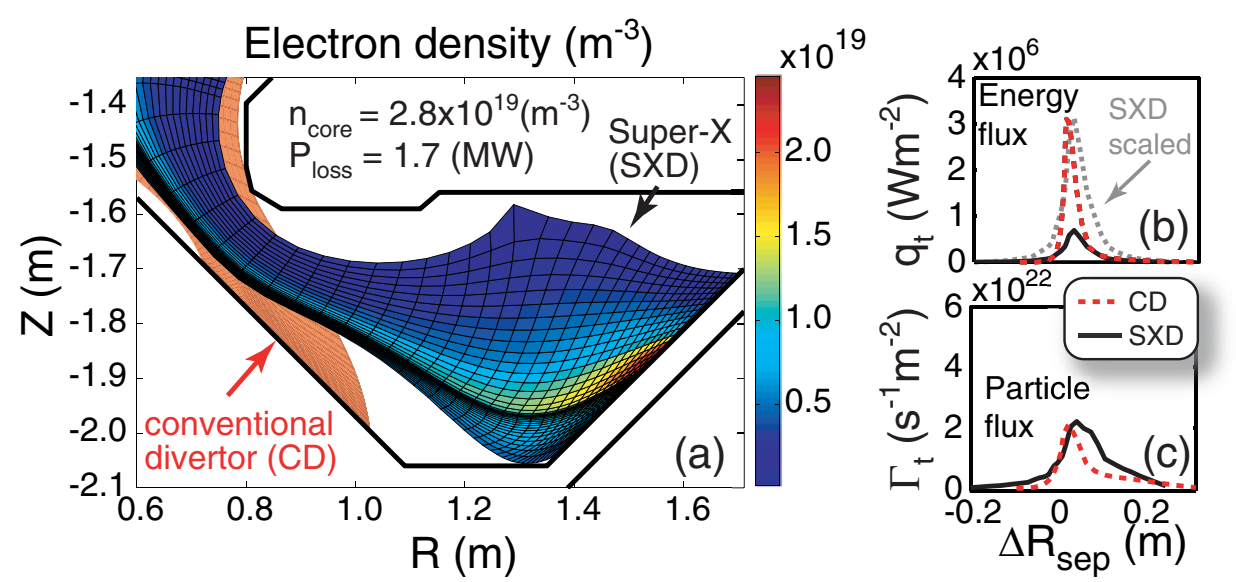

Figure 8. Example SOLPS calculations for MAST Upgrade comparing the conventional divertor (red) with Super-X (black): (a) 2D density, $(b)$ heat and $(c)$ particle flux at the target. Also shown in $(b)$ is a SXD heat flux profile scaled to the maximum of the CD profile (grey, dotted).

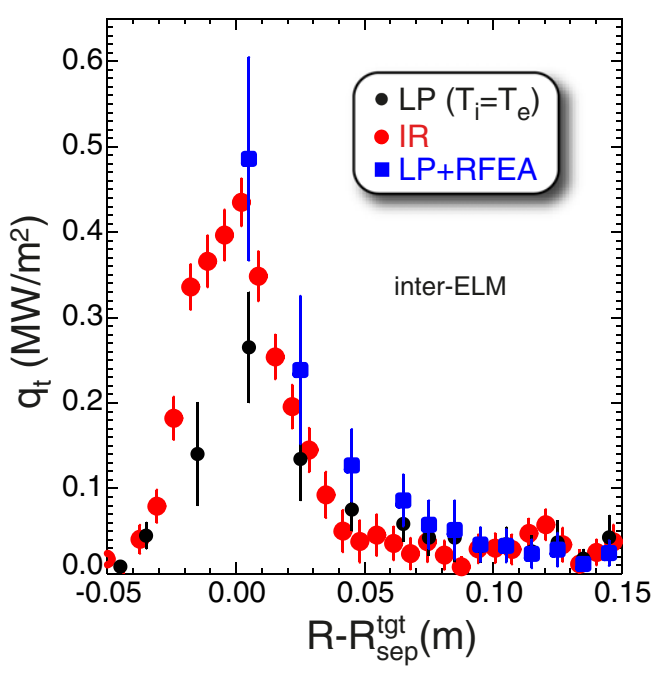

Figure 9. Comparison of heat flux profiles at the lower outer strike point from Langmuir probes (LP, black) assuming $T_{\mathrm{e}}=T_{\mathrm{i}}$, (red) IR camera and (blue) probes using $T_{\mathrm{i}}$ from the divertor RFEA (blue)

(c) the peak particle flux. The SOL both for particles and heat flux is broadened in the SXD case. The SOL heat transport is dominated by conduction leading to a stronger reduction of $T_{\mathrm{e}}$ and $T_{\mathrm{i}}$ at the target in SXD due to the longer connection length. Parameter scans have been performed with SOLPS to test the robustness of specific Upgrade design features. This is necessary to also address uncertainties arising from the ad hoc anomalous diffusivity representing the turbulent cross field (radial) transport in SOLPS. Furthermore, a 1D model has been developed (SOLF1D) and successfully benchmarked against the SOLPS calculation [50]. Here, a strong reduction of heat flux is observed when the divertor leg is stretched to a larger radius or if the recycling source is increased with the SOL volume.

Assumptions not only enter SOL simulations, but also data interpretation. The ion temperature in front of the target plate is one of such assumptions, affecting the interpretation of Langmuir probe measurements. For the first time a retarding field energy analyser (RFEA) has now been used in the down-stream divertor region of MAST to measure $T_{\mathrm{i}}$ close to the target $[47,51,52]$. Figure 9 shows heat flux profiles

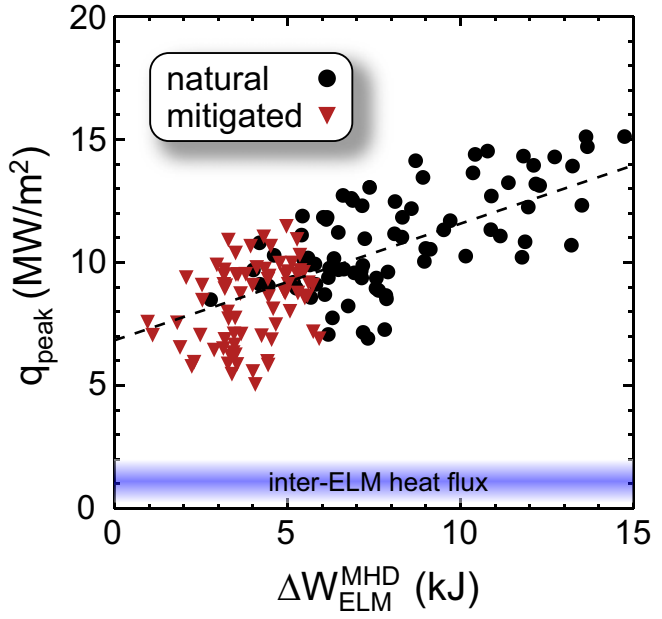

Figure 10. Measured peak heat flux to the target versus ELM energy loss for natural (black dots) and mitigated (red triangles) ELMs. The dashed line is a linear fit to the data.

at the lower outer strike-point during an inter ELM period in a $I_{\mathrm{p}}=0.9 \mathrm{MA}$ type-I ELMy $\mathrm{H}$-mode from Langmuir probes assuming $T_{\mathrm{i}}=T_{\mathrm{e}}$ (black dots), the IR camera (red circles) and Langmuir probe data using $T_{\mathrm{i}}$ from the RFEA measurement (blue squares). The inclusion of the measured ion temperature $T_{\mathrm{i}} / T_{\mathrm{e}} \leqslant 3$ clearly brings the IR and Langmuir probe measurements into closer agreement in the high heat flux region around the strike point, but further out in the SOL leads to an overestimate of the heat flux most pronounced in the region $0.04 \mathrm{~m} \leqslant R-R_{\text {sep }} \leqslant 0.07 \mathrm{~m}$. With increasing collisionality $T_{\mathrm{i}} / T_{\mathrm{e}}$ evolves towards unity as expected. The divertor RFEA measurements are augmented by a similar system at the outer mid-plane, thus allowing the study of the changes of $T_{\mathrm{i}}$ towards the divertor in the SOL for the first time in a tokamak. As shown previously for the pedestal region [37] also in the mid-plane SOL $T_{\mathrm{i}}>T_{\mathrm{e}}$ holds $[51,53]$ agreeing well with edge charge exchange measurements.

These new measurements enhance confidence in the heat load measurements with the IR camera during mitigated and unmitigated ELMs [24, 54]. As can be seen from figure 10 the peak heat flux to the target is reduced for mitigated ELMs 
corresponding to their ELM size, which follows the same $\Delta W \cdot f_{\mathrm{ELM}}=$ const. dependence as natural ELMs [54]. In addition the wetted area decreases with decreasing ELM size as has been observed in other devices. At vanishing ELM size $\left(\Delta W_{\mathrm{ELM}}^{\mathrm{MHD}} \rightarrow 0 \mathrm{~kJ}\right)$ the linearly extrapolated peak heat flux would be much larger than the measured inter ELM heat flux $q_{\mathrm{t}} \approx 0.5-1.5 \mathrm{MW} \mathrm{m}^{-2}$ and a deviation from the linear trend may occur, although MAST data currently show no evidence for this. Strike point spitting during ELM mitigation with RMPs is measured from $\mathrm{C}_{\text {II }}$ narrow band visible imaging in the divertor. These measurements agree well with ERGOS vacuum modelling of the perturbations, though at reduced RMP coil current in ERGOS, implying some screening by the plasma.

Not only the peak heat flux, but also the heat flux profile is important to assess divertor performance in future devices. A database of MAST L- and H-mode discharges has been constructed [55] using a new way of fitting target heat flux profiles developed on JET and ASDEX Upgrade [56] to feed into a multi-machine database [57] and to arrive at a scaling of the heat flux decay length, $\lambda_{q}$, with machine parameters. However, a first principle understanding of the SOL transport processes leading to the profiles of $n_{\mathrm{e}}, T_{\mathrm{e}}$ and $q_{\text {heat }}$ at the target is needed. Many aspects of the L-mode filament dynamics at the mid-plane in MAST seemed to be captured by electrostatic interchange turbulence simulations with ESEL [58,59]. The simulations not only reproduce the ion saturation current mean profile, but also many of the statistical properties of the observed turbulence. Parameter scans of these ESEL simulations have been used to arrive at a scaling for $\lambda_{q}$ at the mid-plane [58]. To make predictions for the target profiles the simple model for the parallel transport in ESEL needs to be improved. Nevertheless, the scaling law, which is in broad agreement to a recent experimental scaling [60], reproduce the expectation that $\lambda_{q}$ does not simply increase with machine size confirming the importance of the forthcoming experimentation in MAST Upgrade [47,58]. In particular it is found that $\lambda_{q} \propto I_{\mathrm{p}}^{-1}$ as experimentally seen on many devices for $\mathrm{L}$ mode [60] and H-mode [57] and increases with the parallel length scale in the SOL, both quantities can be changed in the MAST Upgrade over a large range.

\section{Current drive and fast particle physics}

Current profile, $j(r)$, control and non-inductive current drive are likely needed in future tokamaks and in particular in STs. The JINTRAC integrated suite of codes [61], routinely used for modelling JET discharges, has been interfaced with the MAST data to increase the interpretative and predictive capability beyond TRANSP ${ }^{31}$ [62]. Modelling of the current ramp-up is used as a validation exercise for both codes and previous rampup modelling with TRANSP on MAST [63, 64] and JET [65] suggested a faster modelled current diffusion than observed in the experiment. In the simulations the current diffusion is rather sensitive to errors in the equilibrium reconstruction or experimental mappings. Hence, a set of highly repeatable discharges on MAST with different NBI onset times during the current ramp up to allow MSE measurements within 5 to $10 \mathrm{~ms}$

${ }^{31} \mathrm{http}: / / \mathrm{w} 3 . p p p l . t r a n s p . g o v$.

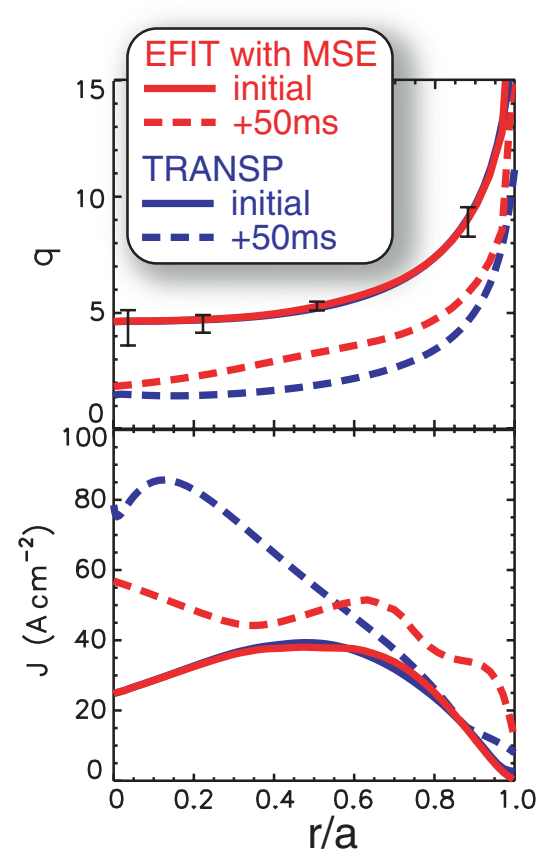

Figure 11. Comparison of the (top) $q$ and (bottom) current density profile evolution during an ohmic current ramp-up with MSE measurements using the NBI onset from a multi shot analysis. Results from (red) an MSE constrained equilibrium reconstruction (EFIT) and (blue) neoclassical poloidal field diffusion (TRANSP) are shown for the (solid) initial profiles and (dashed) profiles after $50 \mathrm{~ms}$ evolution at $\mathrm{d} I / \mathrm{d} t=5 \mathrm{MA} \mathrm{s}^{-1}$ ramp speed.

after beam onset were used to build-up a 'quasi-' ohmic $j(r, t)$ derived from a MSE constrained equilibrium reconstruction on a shot to shot basis [64]. Comparing these new measurements directly with synthetic data from the modelling confirms the previous results of slower $j(r)$ evolution in the experiment (see figure 11).

A key question for future tokamaks is the interplay between fast particle driven instabilities and current drive [66]. Beam ions being super-Alfvénic in STs mimic important aspects of $\alpha$-particles and understanding of neutral beam current drive (NBCD) in the presence of high fast particle pressure is a key aim for the planned Upgrade. The recently installed four channel movable neutron camera (NC) [67] and poloidal and toroidal fast-ion $\mathrm{D}_{\alpha}$ emission diagnostic (FIDA) [68] allow detailed studies of the fast-ion redistribution to be performed already in MAST. In particular the redistribution of fast-ions due to $n=1$ fishbones has been studied in detail $[69,70]$. In figure 12 measured neutron emission profiles for a dedicated set of discharges during $n=1$ fishbone activity are compared with synthetic data of detailed TRANSP runs of the discharges with varying assumptions for the anomalous fast-ion diffusion model in TRANSP with a simple diffusion coefficient uniform in real and velocity space to assess what global level of redistribution may be present. Similar double null discharges (DNDs) with on-axis and single null discharges (SNDs) with off-axis NBI are compared at two different power levels using the same injection energy. In the DND cases an anomalous $\left(D_{\mathrm{FI}}=3 \mathrm{~m}^{2} \mathrm{~s}^{-1}\right)$ redistribution is confirmed by the $\mathrm{NC}$ data at high power, whereas at low power no significant anomalous redistribution is needed to explain the 


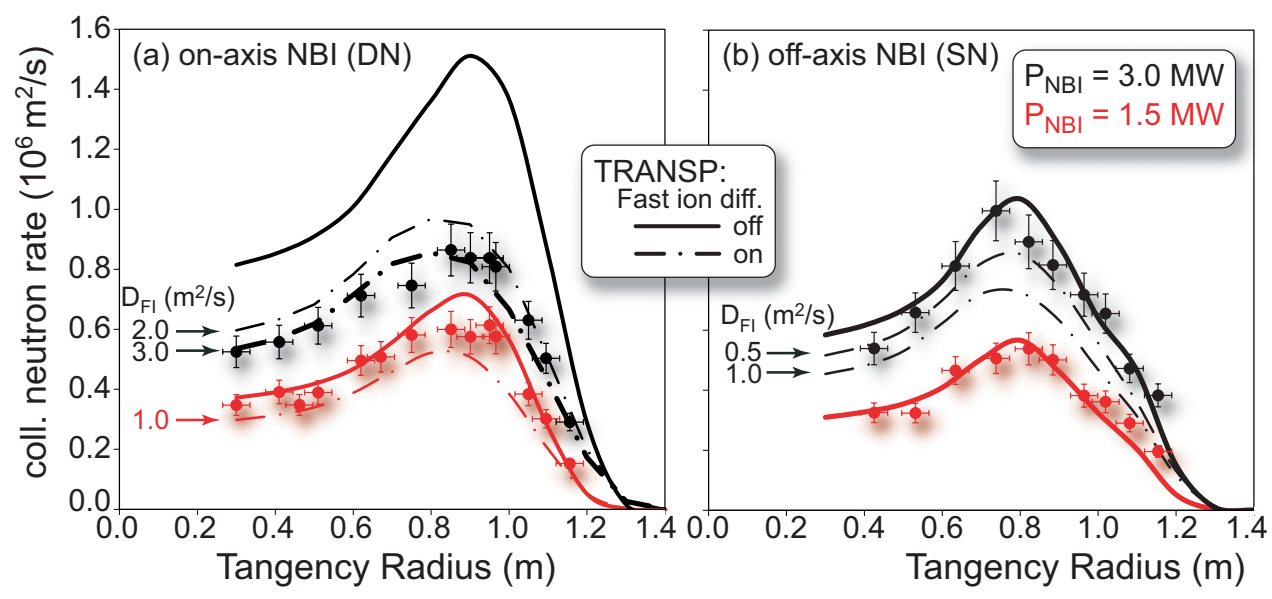

Figure 12. Profiles from the movable neutron camera obtained from a set of similar discharges in comparison with TRANSP run for (a) on-axis and $(b)$ off-axis injection with $1.5 \mathrm{MW}$ (red) and 3.0 MW (black) injected power.

measurements. Here, the higher power causes the $n=1$ fishbone amplitude to increase by more than a factor of two. Off-axis NBI should lead to a reduced fishbone drive even at high power, since the fast-ion pressure gradient in the core is reduced. Indeed in these discharges only weak $n=1$ activity is found and consequently, no fast-ion redistribution is needed to explain the neutron data. The new local and energy resolved FIDA data is consistent with the assumption of $D_{\mathrm{FI}}$ constant in space and energy [71, 72]. However, the data from the poloidal FIDA system seems to suggest that there may be a loss of trapped fast-ions even under conditions where the neutron data can be modelled assuming no anomalous fastion transport. In contrast to the loss of passing fast-ions, which affects the neutron emission strongly, the loss of trapped fast-ions may not produce a significant drop in the neutron rate.

These new data can be compared with HAGIS [73] that links the $n=1$ fishbone mode amplitude to the TRANSP anomalous diffusion coefficient [70]. A highly parallelized new Monte-Carlo full-orbit beam injection code LOCUSTGPU [74], running on dedicated graphics processors, has been developed to calculate smooth representations of the experimental fast-ion distribution function $f_{\mathrm{FI}}=$ $f_{\mathrm{FI}}\left(E, p_{\phi}, \mu ; \sigma\right)$ expressed in terms of constants of motion, to be used directly in the HAGIS modelling extending the calculations using model fast-ion distribution functions presented previously [8]. This, together with the incorporation of collisional drag into the HAGIS modelling of resonant beam ions, has led to an improved understanding of the interactions of these ions with various electromagnetic modes, as well as fast-ion redistribution during fishbones. In particular the inclusion of dynamical friction is important when modelling super-Alfvénic particles. It has been found that the redistribution during fishbones can be expressed as effective diffusion and convection both scaling quadratically with the mode amplitude [70]. However, the transport coefficients computed using HAGIS are not sufficient to account for the drops in neutron emission during fishbone excitation, suggesting that other processes may be contributing to the fast-ion redistribution.

Other mechanisms for fast-ion redistribution and fast-ion losses have also been studied. The ion loss due to the toroidal

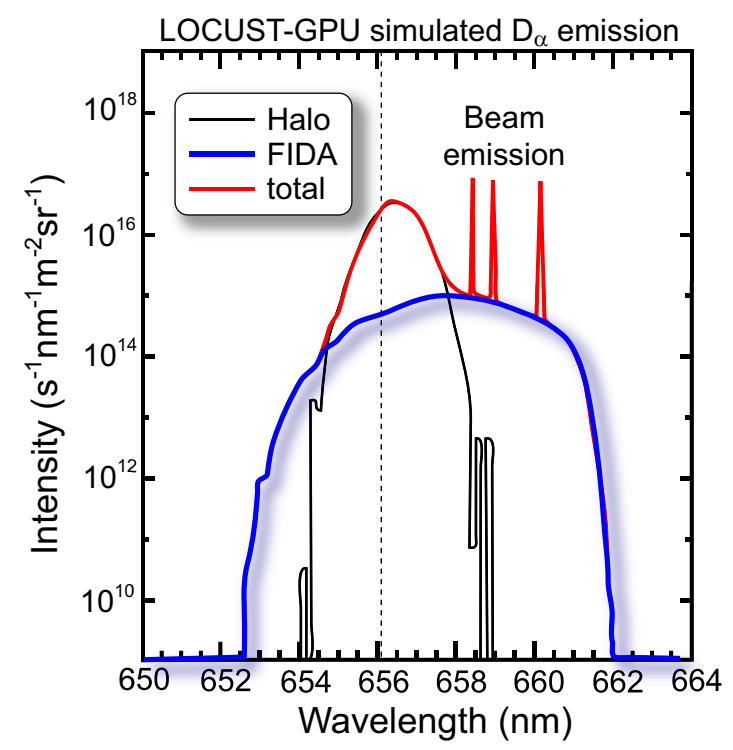

Figure 13. Simulated $\mathrm{D}_{\alpha}$ emission from LOCUST-GPU. FIDA and halo emission result from charge exchange between injected neutrals and, respectively, fast and bulk deuterium ions, while beam emission is produced by the de-excitation of injected neutrals.

field ripple has been modelled with CUEBIT [75] and shown to contribute only $0.1 \mathrm{~m}^{2} \mathrm{~s}^{-1}$ or less to $D_{\mathrm{FI}}$. The changes to the fast-ion distribution caused by sawtooth crashes and an internal $n=1$ kink mode (long-lived mode, LLM) [76] have also been measured with the NC and FIDA [67,72]. During the LLM neutron emission is reduced in the core. The FIDA data are best matched during this phase using a core localized $D_{\mathrm{FI}} \approx 6 \mathrm{~m}^{2} \mathrm{~s}^{-1}$ in contrast to the lower but global redistribution during fishbone activity. Synthetic FIDA and NC diagnostics are embedded in LOCUST-GPU, for better data analysis. An example of the modelled $D_{\alpha}$ spectrum is shown in figure 13.

At the low toroidal field in MAST the high ratio of fast-ion velocity to the Alfvén speed (up to 2.5) leads to the excitation of compressional Alfvén eigenmodes (CAEs) [77] with $n>0$ rotating in the co-current direction. Such modes have to be excited by the anomalous Doppler resonance condition 


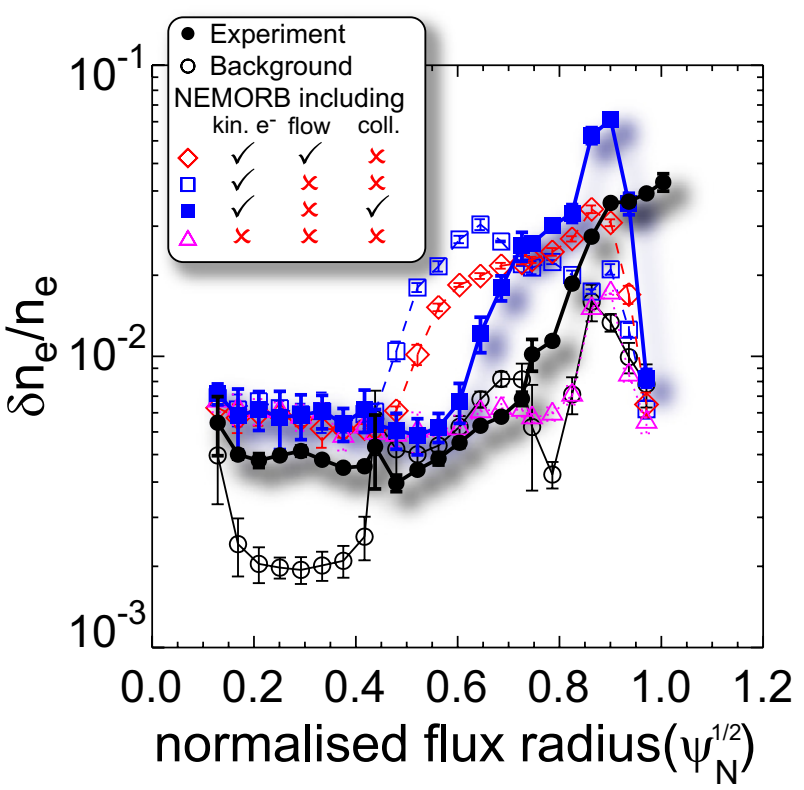

Figure 14. Comparison of (black) BES density fluctuation profiles with synthetic data from NEMORB using different assumptions (see legend).

$\omega-k_{\|} v_{\|}=l \omega_{\mathrm{ci}}$ with $l=-1$ ( $\omega_{\mathrm{ci}}$ : ion cyclotron frequency) and are driven by the beam anisotropy $T_{\|}>T_{\perp}$. Usually CAEs are excited at the normal Doppler resonance $l=+1$ and have $n<0$

\section{Core transport}

In spherical tokamaks ion energy transport is often found to be close to neoclassical, due to the strong perpendicular flow shear stabilizing ITG turbulence [78], except in the periphery of L-mode plasmas where the level of flow shear is too weak [79]. Electron heat transport on the other hand remains strongly anomalous, with one candidate explanation being ETG turbulence, which is less affected by sheared flow [78]. Although in $\mathrm{H}$-mode plasmas in MAST (and indeed also in NSTX) ion-scale turbulence is largely stabilized by $E \times B$ flow shear $[78,79]$, strong ion-scale turbulence is present in the peripheral regions of L-mode plasmas where the level of flow shear is insufficient to fully stabilize this turbulence, this difference being an important feature that distinguishes L- and $\mathrm{H}$-mode plasmas. Otherwise, the core transport properties of $\mathrm{L}-$ and $\mathrm{H}$-mode plasmas are rather similar with anomalous, ion-scale transport being largely stabilized in both cases. This has been confirmed using the new $2 \mathrm{D}(8$ radial $\times 4$ poloidal $)$ BES system [80]. A comparison of BES measurements of L-mode density fluctuations with synthetic data from various NEMORB (global non-linear GK) [79] simulations is shown in figure 14. The level of L-mode turbulence is sensitive to inclusion of: (A) kinetic electrons, (B) collisions and (C) rotation shear (figure 14). The flow shear, as well as all other prescribed profiles in these calculations, has been taken from a detailed TRANSP analysis of the experiment. Inclusion of (A) and (B) brings simulated turbulence amplitude within factor of 2-3 to the experimental data and adding (C) may improve agreement further [81]. Also, at high parallel
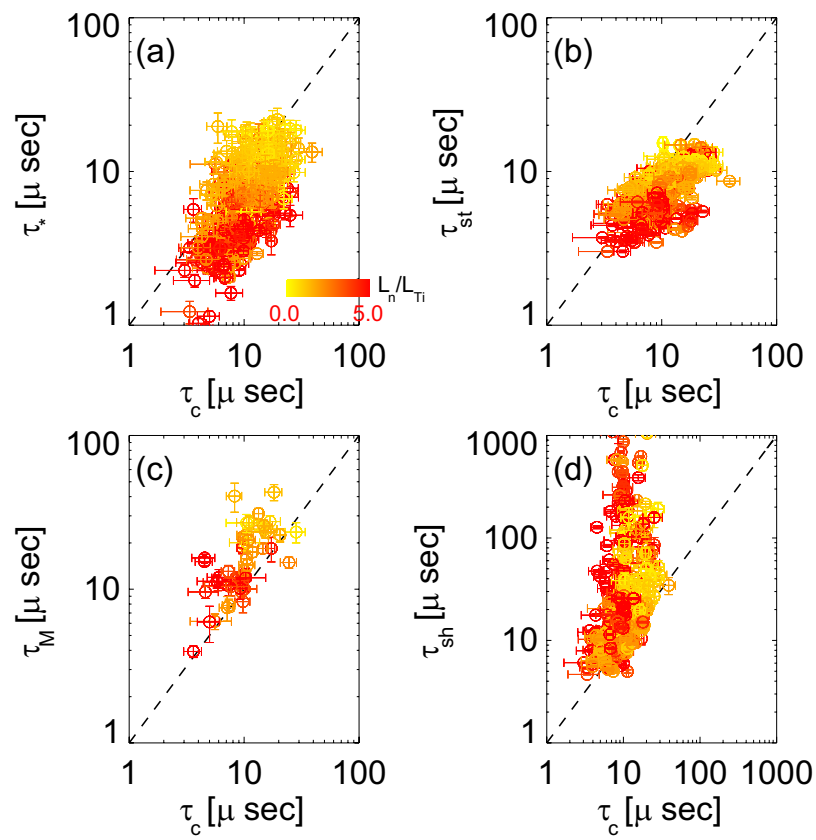

Figure 15. Comparison of turbulence correlation time $\tau_{\mathrm{c}}$ with (a) drift time $\tau_{\star},(b)$ streaming time, $(c)$ magnetic drift time and $(d)$ the perpendicular shearing time.

flow shear subcritical turbulence may cause a finite ion heat flux in regimes where the linear growth rate of the microinstabilities vanishes $[82,83]$. The experimental dependence of $R / L_{T_{\mathrm{i}}}\left(L_{f}^{-1} \equiv \nabla \ln f\right.$ : gradient length) on toroidal flow shear and $q / \epsilon$ is in reasonable agreement with cyclone basecase simulations (a generalized circular s-alpha, tokamak equilibrium, frequently used to benchmark simulations [84]) to obtain the zero-turbulence manifold $[81,83]$.

Turbulence measurements have not only been compared to non-linear GK calculations [81], but also a database of turbulence characteristics has been compiled to perform a statistical comparison to associated linear and non-linear time scales [81,85]. In figure 15 the measured turbulence correlation times $\tau_{\mathrm{c}}$ are compared to $(a)$ the drift time $\tau_{\star}=\left(l_{y} / \rho_{\mathrm{i}}\right) L_{\star} / v_{\mathrm{th}, \mathrm{i}}\left(L_{\star}=\min \left\{L_{T_{\mathrm{i}}}, L_{n}\right\}, l_{y}\right.$ : poloidal correlation length), $(b)$ the parallel streaming time $\tau_{\mathrm{st}}=$ $\left(\pi r / v_{\mathrm{th}, \mathrm{i}}\right) B / B_{p} \sim l_{\|} / v_{\mathrm{th}, \mathrm{i}}\left(B_{p}\right.$ : poloidal field $),(c)$ the magnetic drift time $\tau_{\mathrm{M}}=\left(l_{x} / \rho_{\mathrm{i}}\right) R / v_{\text {th, } \mathrm{i}}$ and $(d)$ the perpendicular velocity shearing time $\tau_{\mathrm{sh}}=\left[\left(B_{p} / B\right) \partial U_{\phi} / \partial r\right]^{-1}\left(U_{\phi}\right.$ : toroidal mean flow). The data show that $\tau_{\mathrm{c}} \sim \tau_{\mathrm{st}} \sim \tau_{\mathrm{M}}$, which indicates a 'grand critical balance' [82] where these basic turbulence characteristics can be predicted from the equilibrium quantities alone. Furthermore, this implies that the parallel and perpendicular correlation length within the flux surface are not independent [85].

It is evident from figure $15(d)$ that for most of the data in our ion-scale turbulence database the $E \times B$ shearing time $\tau_{\mathrm{sh}}$ is longer than the correlation time of the turbulence $\tau_{\mathrm{c}}$. This indicates that where strong ion-scale turbulence is observed, i.e. primarily in the periphery of L-mode plasmas, that the $E \times B$ shearing rate is not the controlling factor governing the de-correlation of the turbulence. It is conjectured in [85] that a significant component of the turbulent amplitude due to zonal flows, which does not contribute to the amplitude of the density 

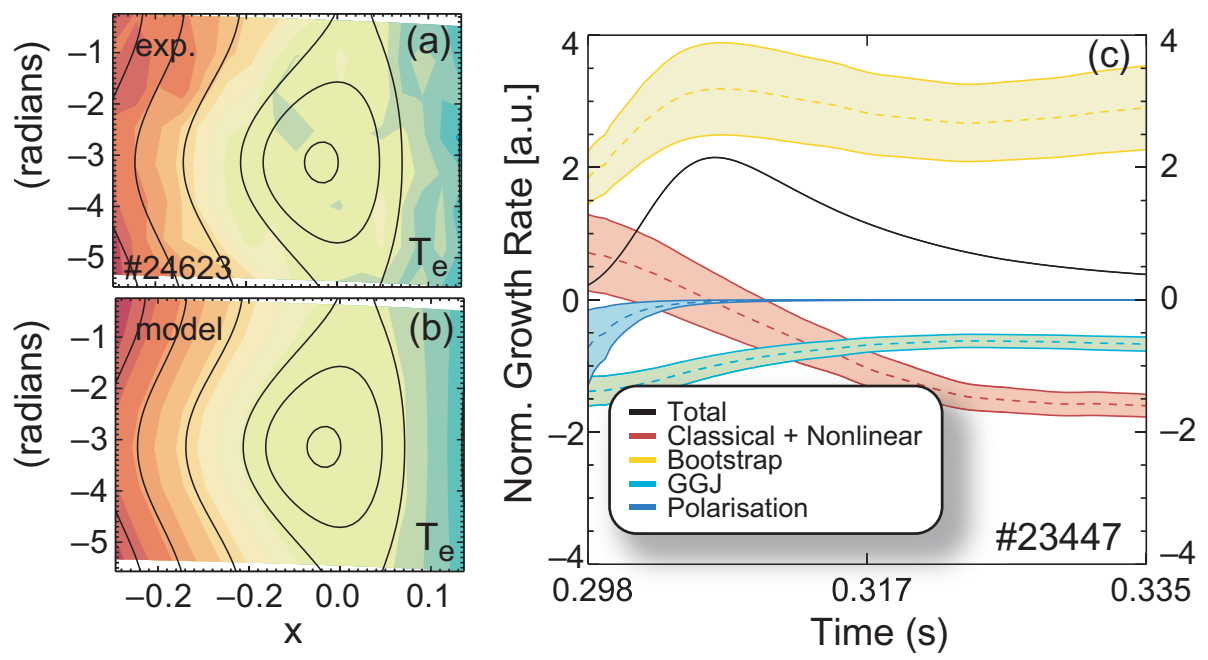

Figure 16. Results of NTM heat flux modelling: left contours of the $(a)$ measured and $(b)$ modelled $T_{\mathrm{e}}$ inside the island (black lines), $(c)$ evolution of terms of the modified Rutherford equation calculated from the modelling, with the classical growth rate including the non-linear classical evolution [86] shown in red.

fluctuations detected by the BES system, may be playing a role in the de-correlation of the turbulence instead.

\section{Core stability}

The NTM is considered as one of the most detrimental modes with respect to confinement and to the probability of disruptions for ITER [87]. In particular modes with $m / n=2 / 1$ helicity ( $m$ poloidal and $n$ toroidal mode number) are the most dangerous. To understand the NTM stability it is important to determine the critical island width, $w_{\mathrm{c}}$, that leads to a flattening of the pressure profile inside the island and thereby the loss of bootstrap current. A common method to determine $w_{\mathrm{c}}$ is to observe the island evolution in experiments where the plasma $\beta$ is ramped down $[88,89]$. Examining the heat transport inside the island, however, is a more fundamental method to understand NTM stability. Using field-programmable gate array (FPGA) based event triggering of the Thomson scattering system $[90,91]$ the $T_{\mathrm{e}}$ and $n_{\mathrm{e}}$ profiles at eight time slices through the rotating $2 / 1$ island of an NTM have been measured for the first time in an ST [92]. A 2D simple heat transport model assuming divergence-free diffusive heat transport in the island with constant parallel and perpendicular heat diffusivity has been used to fit to the $T_{\mathrm{e}}$ profile. The model has been validated against the island width estimation during $\beta$ ramps. Figures $16(a)$ and $(b)$ show the good comparison between experimental and modelled $T_{\mathrm{e}}$ profiles. From the modelling of an ensemble of similar discharges with saturated NTMs the different terms in the modified Rutherford equation have been estimated with errors resulting from the measurement errors (see figure 16(c)) with the most unstable term being the bootstrap drive and all other terms small and stabilizing. A key feature of this analysis is that no specific models for parallel or perpendicular heat transport are assumed, which has not been the case in many previous analyses of NTM growth. The same technique of intelligent event triggering can be used to mitigate the NTM in H-mode by shifting the plasma briefly of DND triggering a short L-mode transition [36] leading to a temporary reduction of $\beta_{\mathrm{p}}$ [91].

Disruption avoidance and mitigation are key features for ITER due to the large forces and the potential for runaway electron generation. On MAST a systematic study of disruption mitigation by massive gas injection with respect to gas load and impurity species $[47,93,94]$ supports findings on other devices. The peak energy load can be reduced by $60 \%$ compared to unmitigated disruptions and the best results with respect to penetration speed and thermal quench time are achieved with an $\operatorname{Ar}(10 \%) / \mathrm{He}$ mix. The relative reduction of energy is found to increase with increasing stored energy.

\section{EBW imaging and other technical improvements}

One of the most exciting novel diagnostics on MAST is the electron Bernstein emission synthetic aperture imaging $[95,96]$, which measures the emission window in poloidal and toroidal angle at $\Delta t \approx 10 \mu$ s time resolution with a $10 \mathrm{MHz}$ sampling rate. This is achieved by using an array of eight antennas to synthesize a 2D image and 16 frequencies for probing at different plasma densities (radial positions) giving 3D information of the EBW emission. Figure 17(a) shows the modelled EBW emission using a 1D full wave modecoupling code complemented with 3D ray-tracing [97] and figure $17(b)$ the corresponding measurement. The angular positions of the emission cones show excellent agreement, with the vertical elongation in the experimental data caused by the shape of the antenna array, and should allow ELMresolved measurements of $j(r)$ in the steep gradient region of the pedestal. The system simultaneously also records active data using one of the antennas as the emitter and recording the reflected power similar to Doppler back scattering, providing density turbulence and velocity fluctuation measurements. A 2D image of the turbulent plasma flows reconstructed from the Doppler shift of the reflected signal is shown in figure $17(c)$. The analysis of the large amount of data is ongoing, but the first results are promising with respect to flows and ELM-resolved edge current densities. 


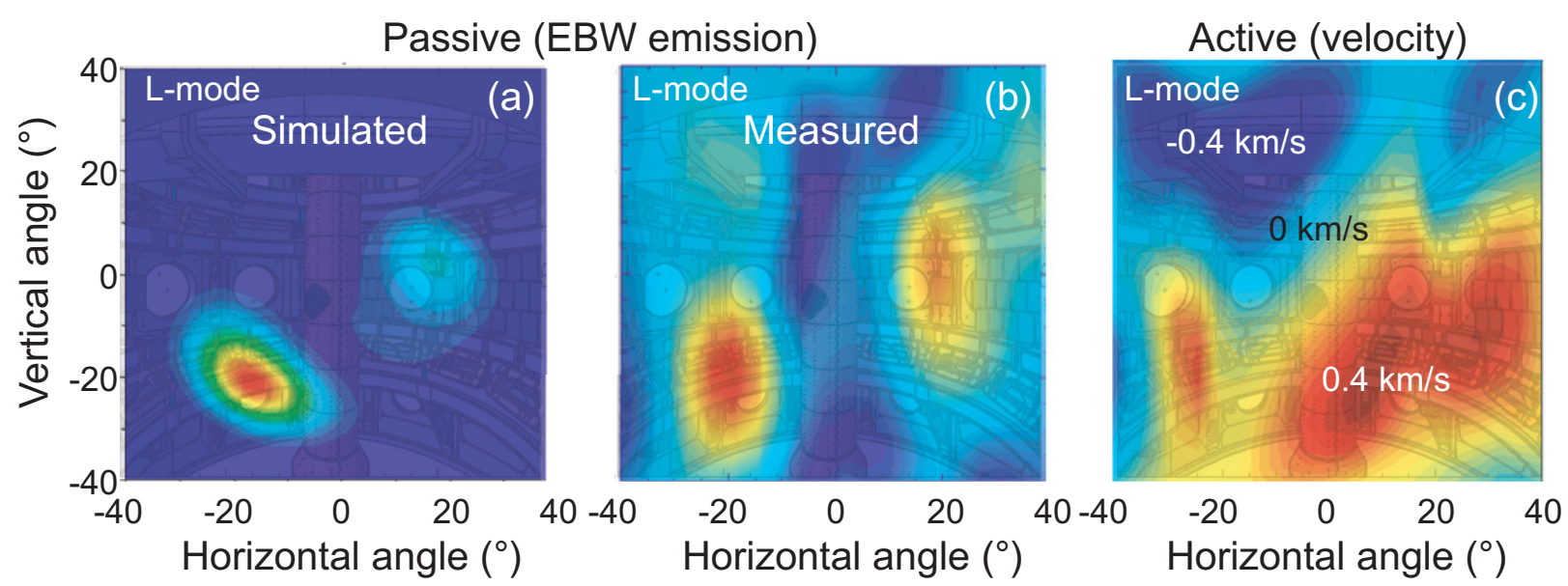

Figure 17. Initial results of the EBW imaging system in L-mode showing $(a)$ the modelled and $(b)$ the measured EBW emission as well as (c) plasma flows from active probing.

As several other systems on MAST this diagnostic is also driven by FPGAs and the application of FPGAs in fusion technology on MAST is growing with development and individual programming done in-house. Apart from the event triggering, already mentioned, an intelligent drive of the TAE antenna has been developed to stop the drive power briefly whilst measuring the plasma response and a system to improve the vertical feedback control is in development to be used on MAST Upgrade.

New piezo-electric valve mechanisms were developed for the HFS mid-plane gas fuelling and the movable gas nozzle. The former, currently operated with a solenoid drive, now enables the mid-plane HFS fuelling to be shut off, thus avoiding the low pedestal temperatures prevalent with continuous fuelling. Here, the valve mechanism is extended from the outside of the vessel to provide sealing close to the plasma. This gives similar fuelling rates to the old system at ten times lower gas pressure and has enabled disruption mitigation studies with massive gas injection from the high field side. The latter allows a modulated gas influx with up to $500 \mathrm{~Hz}$ close to the plasma with response times $<1 \mathrm{~ms}$ and fuelling rates of the order of $10^{21}$ particles $\mathrm{s}^{-1}$ e.g. for impurity transport studies. Both valve systems act as prototypes for the fuelling system design on the MAST Upgrade. In particular the HFS mid-plane fuelling location is foreseen to be the main fuelling location on the Upgrade and needs to be able to support density feedback.

\section{Summary}

MAST continues to make important contributions to ITER/DEMO physics and the general understanding of tokamak plasmas as well as building the physics basis for future spherical tokamak (ST) applications on the way to fusion power. New and sometimes unique measurements such as electron Bernstein wave imaging are fundamental to the progress. The Upgrade (under procurement) will further strengthen these research capabilities and recent studies have aided the design and clarified the physics capabilities. In particular the new divertor design in the MAST Upgrade will help to address the critical exhaust issue, which is even more challenging in the compact ST geometry. Initial modelling suggests that a strong reduction in target heat flux in the Upgrade may be accomplished with a radially extended outer leg.

On MAST mitigation of edge localized modes (ELMs) using resonant magnetic perturbations with $n=3,4,6$ has been demonstrated with the increase in ELM frequency likely to be caused by the 3D deformation of the plasma, and has been shown to be compatible with pellet fuelling. The peak heat flux during an ELM is found to be proportional to the ELM energy loss with mitigated ELMs following the same trend as natural ELMs. At low collisionality the ion temperature in the divertor, measured using a retarding field energy analyser, exceeds the electron temperature showing that the usual assumption of equality between these temperatures for probe data analysis is not always justified. The measured edge velocity fluctuations during an oscillatory phase preceding the formation of the edge transport barrier ( $\mathrm{L}-\mathrm{H}$ transition) raise questions for simple predator-prey-like dynamics between the turbulence driven flow shear and turbulence suppression by the flow shear. Core ion-scale turbulence in L-mode has been measured to be consistent with a 'grand-critical-balance', where the characteristic turbulent time scales are of the same order of magnitude. Global gyro-kinetic modelling shows reasonable agreement with the measured fluctuation amplitude over the full plasma radius, when kinetic electron effects and collisions are taken into account. Measurements of the 2D temperature profile inside an island due to a neoclassical tearing mode enable a detailed analysis of the island evolution without assuming a specific model for the parallel and perpendicular heat transport inside the island. The fast-ion redistribution by fishbones is found to be low with off-axis neutral beam injection. This bodes well for the MAST Upgrade and its planned off-axis heating as well as for future ST devices.

\section{Acknowledgments}

This work was funded by the RCUK Energy Programme under grant EP/I501045 and the European Communities under the contract of Association between EURATOM and CCFE. The views and opinions expressed herein do not necessarily reect those of the European Commission.

(c) Euratom 2013 


\section{References}

[1] Darke A. et al 1994 MAST: a MEGA AMP spherical tokamak 18th Symp. on Fusion Technology (Karlsruhe, Germany, 22-26 August 1994)

[2] Ono M. et al 2001 Overview of initial NSTX experimental results Nucl. Fusion 41 1435-47

[3] Shimada M. et al 2007 Progress in the ITER physics basis: chapter 1. Overview and summary Nucl. Fusion $47 \mathrm{~S} 1$

[4] Hender T.C., Voss G.M. and Taylor N.P. 1999 Spherical tokamak volume neutron source Fusion Eng. Des. 45 265-79

[5] Peng Y-.K.M. et al 2005 A component test facility based on the spherical tokamak Plasma Phys. Control. Fusion 47 B263

[6] Voss G.M., Davis S., Dnestrovskij A., Kirk A., Knight P.J., Loughlin M., O’Brien M.H., Sychugov D., Tabasso A. and Wilson H.R. 2008 Conceptual design of a component test facility based on the spherical tokamak Proc. 8th Int. Symp. Fusion Nuclear Technology-ISFNT-8 SI (Heidelberg, Germany, 30 September-5 October 2007); Fusion Eng. Des. 83 1648-53 http://www.sciencedirect.com/science/article/B6V3C4SXRTPV-1/2/1e43fa1c7a7d5ea0b2c7d01297b182c0

[7] Meyer H. et al, the MAST and NBI Teams 2009 Overview of physics results from MAST Nucl. Fusion 49104017

[8] Lloyd B. et al, the MAST and NBI Teams 2011 Overview of physics results from MAST Nucl. Fusion 51094013

[9] Stork D. et al 2010 The upgrade to the MEGA AMP Spherical Tokamak Proc. 23rd Int. Conf. on Fusion Energy 2010 (Daejeon, Korea, 2010) (Vienna: IAEA) CD-ROM file ICC/P5-06 and www-naweb.iaea.org.uk/napc/physics/FEC/ FEC2010/html/index.htm

[10] Morris A.W. 2012 MAST: results and upgrade activities IEEE Trans. Plasma Sci. 40 682-91

[11] Valanju P.M., Kotschenreuther M., Mahajan S.M. and Canik J. 2009 Super-X divertors and high power density fusion devices Phys. Plasmas 16056110

[12] Ryutov D D 2007 Geometrical properties of a 'snowflake' divertor Phys. Plasmas 14064502

[13] Snyder P.B., Groebner R.J., Leonard A.W., Osborne T.H. and Wilson H.R. 2009 Development and validation of a predictive model for the pedestal height Phys. Plasmas 16056118

[14] Scannell R. et al 2012 Dynamics of the plasma edge during the $\mathrm{L}-\mathrm{H}$ transition and $\mathrm{H}$-mode in MAST(EXC/P7-22) 24th IAEA Fusion Energy Conf. (San Diego, CA, 8-13 October 2012) www-naweb.iaea.org/napc/physics/FEC/FEC2012/ html/proceedings.pdf

[15] Dickinson D., Saarelma S., Scannell R., Kirk A., Roach C.M. and Wilson H.R. 2011 Towards the construction of a model to describe the inter-ELM evolution of the pedestal on MAST Plasma Phys. Control. Fusion 53115010

[16] Dickinson D., Roach C.M., Saarelma S., Scannell R., Kirk A. and Wilson H.R. 2012 Kinetic instabilities that limit $\beta$ in the edge of a tokamak plasma: a picture of an H-mode pedestal Phys. Rev. Lett. 108135002

[17] Kotschenreuther M., Rewoldt G. and Tang W.M. 1995 Comparison of initial value and eigenvalue codes for kinetic toroidal plasma instabilities Comput. Phys. Commun. 88 128-40

[18] Roach C.R. et al 2012 Gyrokinetic instabilities in an evolving tokamak H-mode (TH/5-1) 24th IAEA Fusion Energy Conf. (San Diego, CA, 8-13 October 2012) www-naweb.iaea.org/ napc/physics/FEC/FEC2012/html/proceedings.pdf

[19] Saarelma S. et al 2012 Pedestal modelling based on ideal MHD and gyrokinetic stability analyses on JET and ITER plasmas (TH/P3-10) 24th IAEA Fusion Energy Conf. (San Diego, CA, 8-13 October 2012) www-naweb.iaea.org/ napc/physics/FEC/FEC2012/html/proceedings.pdf

[20] Loarte A. et al 2007 Transient heat loads in current fusion experiments, extrapolation to ITER and consequences for its operation Phys. Scr. T128 222-8
[21] Evans T.E. et al 2006 Edge stability and transport control with resonant magnetic perturbations in collisionless tokamak plasmas Nature Phys. 2 419-23

[22] Nardon E. et al 2009 ELM control by resonant magnetic perturbations on JET and MAST Proc. 18th Int. Conf. on Plasma-Surface Interactions in Controlled Fusion Device (Toledo, Spain, 26-30 May 2008); J. Nucl. Mater.

390-391 773-6 http://www.sciencedirect.com/ science/article/B6TXN-4VG5J11-Y/2/ f1272701e04e2fbcf85b080dafcc1b36

[23] Kirk A. et al and the MAST Team 2010 Resonant magnetic perturbation experiments on MAST using external and internal coils for ELM control Nucl. Fusion 50034008

[24] Kirk A. et al 2012 Understanding ELM mitigation by resonant magnetic perturbations on MAST(EX/3-2) 24th IAEA Fusion Energy Conf. (San Diego, CA, 8-13 October 2012) www-naweb.iaea.org/napc/physics/FEC/FEC2012/html/ proceedings.pdf

[25] Liu Y., Kirk A. and Nardon E. 2010 Full toroidal plasma response to externally applied nonaxisymmetric magnetic fields Phys. Plasmas 17122502

[26] Liu Y., Kirk A., Gribov Y., Gryaznevich M.P., Hender T.C. and Nardon E. 2011 Modelling of plasma response to resonant magnetic perturbation fields in MAST and ITER $\mathrm{Nucl}$. Fusion 51083002

[27] Kirk A., Harrison J., Liu Y., Nardon E., Chapman I.T. and Denner P. 2012 Observation of lobes near the X point in resonant magnetic perturbation experiments on MAST Phys. Rev. Lett. 108255003

[28] Bécoulet M. et al 2008 Numerical study of the resonant magnetic perturbations for type I edge localized modes control in ITER Nucl. Fusion 48024003

[29] Cooper W.A., Hirshman S.P., Merkel P., Graves J.P., Kisslinger J., Wobig H.F.G., Narushima Y., Okamura S. and Watanabe K.Y. 2009 Three-dimensional anisotropic pressure free boundary equilibria Comput. Phys. Commun. 180 1524-33

[30] Chapman I.T., Kirk A., Saarelma S., Harrison J.R., Scannell R. and the MAST Team 2012 Towards understanding ELM mitigation: the effect of axisymmetric lobe structures near the X-point on ELM stability Nucl. Fusion 52123006

[31] Valovič M., Cunningham G., Garzotti L., Gurl C., Kirk A., Naylor G., Patel A., Scannell R., Thornton A.J. and the MAST Team 2013 Pellet fuelling of plasmas with edge localized modes mitigation by resonant magnetic perturbations in MAST Plasma Phys. Control. Fusion 55025009

[32] Kukushkin A.S., Polevoi A.R., Pacher H.D., Pacher G.W. and Pitts R.A. 2011 Physics requirements on fuel throughput in ITER Proc. 19th Int. Conf. on Plasma-Surface Interactions in Controlled Fusion (San Diego, CA, USA, 24-28 May 2010) J. Nucl. Mater. 415 (Suppl.) S497-500 http://www. sciencedirect.com/science/article/pii/S0022311510004411

[33] Fenstermacher M.E. et al 2010 ELM control by resonant magnetic perturbations: overview of research by the PEP ITPA group Technical Report EFDA-JET-CP(10)08/31, EFDA-JET

[34] Valovič M., Garzotti L., Gurl C., Akers R., Harrison J., Michael C., Naylor G., Scannell R. and the MAST Team 2012 H-mode access by pellet fuelling in the MAST tokamak Nucl. Fusion 52114022

[35] Meyer H., Carolan P.G., Conway N.J., Counsell G.F., Cunningham G., Field A.R., Kirk A., McClements K.G., Price M., Taylor D. and the MAST Team 2005 Improved $\mathrm{H}$-mode access in connected DND in MAST Plasma Phys. Control. Fusion 47 843-67

[36] Meyer H., Andrew Y., Carolan P.G., Cunningham G., Delchambre E., Field A.R., Kirk A., Molchanov P. Rozhansky V., Voskoboynikov S. and MAST and NBI Teams 2008 Active control of the H-mode transition on MAST Plasma Phys. Control. Fusion 50015005 
[37] Meyer H. et al, the MAST and NBI Teams 2011 L-H transition and pedestal studies on MAST Nucl. Fusion 51113011

[38] Conway G.D., Angioni C., Ryter F., Sauter P. and Vicente J. 2011 Mean and oscillating plasma flows and turbulence interactions across the $\mathrm{L}-\mathrm{H}$ confinement transition Phys. Rev. Lett. 106065001

[39] Schmitz L., Zeng L., Rhodes T.L., Hillesheim J.C., Doyle E.J., Groebner R.J., Peebles W.A., Burrell K.H. and Wang G. 2012 Role of zonal flow predator-prey oscillations in triggering the transition to H-mode confinement Phys. Rev. Lett. 108155002

[40] Lotka A.J. 1910 Contribution to the theory of periodic reaction J. Phys. Chem. 14 271-4

[41] Leboeuf J.-N., Charlton L.A. and Carreras B.A. 1993 Shear flow effects on the nonlinear evolution of thermal instabilities Phys. Fluids B 5 2959-66

[42] Miki K. and Diamond P.H. 2011 Novel states of pre-transition edge turbulence emerging from shearing mode competition Nucl. Fusion 51103003

[43] Robinson J.R., Hnat B., Dura P., Kirk A., Tamain P. and the MAST Team 2012 Interaction between a low-frequency electrostatic mode and resonant magnetic perturbations in MAST Plasma Phys. Control. Fusion 54105007

[44] LaBombard B. et al and the Alcator C-Mod Team 2005 Transport-driven scrape-off layer flows and the X-point dependence of the $\mathrm{L}-\mathrm{H}$ power threshold in Alcator C-Mod Phys. Plasmas 12056111

[45] Canik J.M., Maingi R., Soukhanovskii V.A., Bell R.E., Kugel H.W., LeBlanc B.P. and Osborne T.H. 2011 Measurements and 2-D modeling of recycling and edge transport in discharges with lithium-coated PFCs in NSTX Proc. 19th Int. Conf. on Plasma-Surface Interactions in Controlled Fusion (San Diego, CA, USA, 24-28 May 2010); J. Nucl. Mater. 415 (Suppl.) S409-12 http://www. sciencedirect.com/science/article/pii/S0022311510007920

[46] Matthews G.F. 2013 Plasma operation with an all metal first-wall: comparison of an ITER-like wall with a carbon wall in JET J. Nucl. Mater. 438 S2-10

[47] Fishpool G. et al 2012 MAST contributions to the exhaust challenge, including testing of Super-X (EX/P5-17) 24th IAEA Fusion Energy Conf. (San Diego, CA, 8-13 October 2012) www-naweb.iaea.org/napc/physics/FEC/ FEC2012/html/proceedings.pdf

[48] Fishpool G., Canik J., Cunningham G., Harrison J., Katramados I., Kirk A., Kovari M., Meyer H. and Scannell R. 2013 MAST-Upgrade divertor facility and assessing performance of long-legged divertors $\mathrm{J}$. Nucl. Mater. 438 S356-9

[49] Bonnin X., Coster D., Pitcher C.S., Schneider R., Reiter D., Rozhansky V., Voskoboynikov S. and Bürbaumer H. 2003 Improved modelling of detachment and neutral-dominated regimes using the SOLPS B2-Eirene code Proc. 15th Int. Conf. on Plasma-Surface Interactions in Controlled Fusion (Gifu, Japan, 26-31 May 2002); J. Nucl. Mater.

313-316 909-13 http://www.sciencedirect.com/science/ article/pii/S0022311502014757

[50] Havlíčková E., Fundamenski W., Wischmeier M., Fishpool G. and Coster D. 2013 Numerical studies of effects associated with the Super-X divertor on target parameters in MAST-U J. Nucl. Mater. 438 S545-9

[51] Elmore S., Allan S.Y., Kirk A., Fishpool G., Harrison J., Tamain P., Kočan M., Gaffka R., Stephen R., Bradley J.W. and the MAST Team 2012 Upstream and divertor ion temperature measurements on MAST by retarding field energy analyser Plasma Phys. Control. Fusion 54065001

[52] Elmore S., Allan S.Y., Kirk A., Thornton A.J., Harrison J.R., Tamain P., Kočan M. and Bradley J.W. 2013 Scrape-off layer ion temperature measurements at the divertor target in MAST by retarding field energy analyser J. Nucl. Mater. 438 S $1212-5$
[53] Allan S.Y., Elmore S., Kirk A., Kočan M. and Tamain P. 2013 Ion energy measurements on MAST using a midplane RFEA J. Nucl. Mater. 438 S530-5

[54] Thornton A.J., Kirk A., Chapman I.T. and Harrison J.R. 2013 Divertor heat fluxes and profiles during mitigated and unmitigated edge localised modes (ELMs) on the Mega Amp Spherical Tokamak (MAST) J. Nucl. Mater. 438 S199-202

[55] Harrison J. et al 2012 L-mode and inter-ELM divertor particle and heat flux width scaling on MAST 20th Int. Conf. on Plasma Surface Interactions (Aachen, Germany, 2012)

[56] Eich T., Sieglin B., Scarabosio A., Fundamenski W., Goldston R.J. and Herrmann A. 2011 Inter-ELM power decay length for JET and ASDEX Upgrade: measurement and comparison with Heuristic drift-based model Phys. Rev. Lett. 107215001

[57] Eich T. et al 2012 Scaling of the tokamak near scrape-off layer $\mathrm{H}$-mode power width and implications for ITER (ITR/1-1) 24th IAEA Fusion Energy Conf. (San Diego, CA, 8-13 October 2012) www-naweb.iaea.org/napc/physics/FEC/ FEC2012/html/proceedings.pdf

[58] Militello F., Tamain P., Fundamenski W., Kirk A., Naulin V., Nielsen A.H. and the MAST Team 2013 Experimental and numerical characterization of the turbulence in the scrape-off layer of MAST Plasma Phys. Control. Fusion 55025005

[59] Militello F., Fundamenski W., Naulin V. and Nielsen A.H. 2013 Numerical investigation of scrape off layer anomalous particle transport for MAST parameters J. Nucl. Mater. 438 S530-5

[60] Scarabosio A., Eich T., Herrmann A. and Sieglin B. 2013 Outer target heat fluxes and power decay length scaling in L-mode plasmas at JET and AUG Proc. 20th Int. Conf. on Plasma-Surface Interactions in Controlled Fusion Devices (Aachen, Germnay, 20-25 May 2012); J. Nucl. Mater. 438 (Suppl.) S426-30 http://www.sciencedirect.com/ science/article/pii/S0022311513000949

[61] Wiesen S. et al 2008 JINTRAC-JET modelling suite JET ITC-Report EFDA-JET

[62] Hawryluk R.J. 1980 An emperical approach to tokamak transport Physics of Plasmas Close to Thermonuclear Conditions vol 1 ed B. Coppi et al (Brussels: CEC)

[63] Keeling D., Akers R., Challis C.D., Cunningham G. and Meyer H. 2008 Tayloring the $q$-profile on MAST for scenario optimisation 35th EPS Plasma Physics Conf. (Hersonissos, Greece, 9-13 June 2008) http://epsppd.epfl.ch/Hersonissos/start.html

[64] Keeling D., Akers R.J., de Bock M.F.M., Challis C.D., Michael A., Patel C.A. and the MAST Team 2011 Test of current diffusion modelling in MAST current ramp-up 38th EPS Plasma Physics Conf. (Strasbourg, France, 27 June-1 July 2011)

[65] Jenkins I., Baruzzo M., Brix M., Challis C.D., Hawkes N.C., Litaudon J., Mailloux X., Rimini F.G., de Vries P.C. and JET EFDA Contributors 2010 Test of current ramp modelling for AT regimes in JET 37th EPS Plasma Physics Conf. (Dublin, Ireland, 21-25 June 2010)

[66] Turnyanskiy M., Keeling D.L., Akers R.J., Cunningham G., Conway N.J., Meyer H., Michael C.A. and Pinches S.D. 2009 Study of the fast ion confinement and current profile control on MAST Nucl. Fusion 49065002

[67] Cecconello M., Sangaroon S., Turnyanskiy M., Conroy S., Wodniak I., Akers R.J., Ericsson G. and the MAST Team 2012 Observation of fast ion behaviour with a neutron emission profile monitor in MAST Nucl. Fusion $\mathbf{5 2} 094015$

[68] Michael C.A. et al 2013 Dual view FIDA measurements on MAST Plasma Phys. Control. Fusion 55095007

[69] Turnyanskiy M., Challis C.D., Akers R.J., Cecconello M., Keeling D.L., Kirk A., Lake R., Pinches S.D., Sangaroon S. and Wodniak I. 2013 Measurement and control of the fast ion redistribution on MAST Nucl. Fusion 53053016 
[70] Pinches S.D. et al 2012 Development of a predictive capability for fast ion behaviour in MAST(TH/P3-34) 24th IAEA Fusion Energy Conf. (San Diego, CA, 8-13 October 2012) www-naweb.iaea.org/napc/physics/FEC/FEC2012/html/ proceedings.pdf

[71] Jones O.M., Michael C.A., McClements K.G., Conway N.J., Crowley B., Akers R.J., Lake R.J., Pinches S.D. and the MAST team 2013 Fast-ion deuterium alpha spectroscopic observations of the effects of fishbones in the Mega-Ampere Spherical Tokamak Plasma Phys. Control. Fusion $\mathbf{5 5} 085009$

[72] Michael C.A. et al 2013 Dual view FIDA measurements on MAST Plasma Phys. Control. Fusion 55095007

[73] Pinches S.D. et al 1998 The HAGIS self-consistent nonlinear wave-particle interaction model Comput. Phys. Commun. 111 133-49

[74] Akers R. et al 2012 GPGPU Monte Carlo calculation of gyro-phase resolved fast ion and $n$-state resolved neutral deuterium distributions 39th EPS Plasma Physics Conf. (Stockholm, Sweden, 2-6 June 2012) p P5.131

[75] McClements K.G. and Hole M.J. 2012 Toroidal ripple transport of beam ions in the mega-ampère spherical tokamak Phys. Plasmas 19072514

[76] Chapman I.T., Hua M.-D., Pinches S.D., Akers R.J., Field A.R., Graves J.P., Hastie R.J., Michael C.A. and the MAST Team 2010 Saturated ideal modes in advanced tokamak regimes in MAST Nucl. Fusion 50045007

[77] Gorelenkov N.N., Fredrickson E.D., Heidbrink W.W., Crocker N.A., Kubota S. and Peebles W.A. 2006 Discrete compressional Alfvén eigenmode spectrum in tokamaks Nucl. Fusion 46 S933

[78] Roach C.M. et al 2009 Gyrokinetic simulations of spherical tokamaks Plasma Phys. Control. Fusion 51124020

[79] Saarelma S., Hill P., Bottino A., Colyer G., Field A.R., McMillan B., Peeters A., Roach C.M. and the MAST Team 2012 Global gyrokinetic turbulence simulations of MAST plasmas Plasma Phys. Control. Fusion 54085012

[80] Field A.R., Dunai D., Gaffka R., Ghim Y.c., Kiss I., Meszaros B., Krizsanoczi T., Shibaev S. and Zoletnik S. 2012 Beam emission spectroscopy turbulence imaging system for the MAST spherical tokamak Rev. Sci. Instrum. 83013508

[81] Field A.R. et al 2012 Characterisation of ion scale turbulence in MAST(EXC/P-129) 24th IAEA Fusion Energy Conf. (San Diego, CA, 8-13 October 2012) www-naweb.iaea.org/ napc/physics/FEC/FEC2012/html/proceedings.pdf

[82] Barnes M., Parra F.I., Highcock E.G., Schekochihin A.A., Cowley S.C. and Roach C.M. 2011 Turbulent transport in tokamak plasmas with rotational shear Phys. Rev. Lett. 106175004

[83] Highcock E.G., Schekochihin A.A., Cowley S.C., Barnes M., Parra F.I., Roach C.M. and Dorland W. 2012 Zero-turbulence manifold in a toroidal plasma Phys. Rev. Lett. 109265001

[84] Waltz R.E. and Miller R.L. 1999 Ion temperature gradient turbulence simulations and plasma flux surface shape Phys. Plasmas 6 4265-71
[85] Ghim Y.-c., Schekochihin A.A., Field A.R., Abel I.G., Barnes M., Colyer G., Cowley S.C., Parra F.I., Dunai D. and Zoletnik S. 2013 Experimental signatures of critically balanced turbulence in MAST Phys. Rev. Lett. 110145002

[86] Hastie R.J., Militello F. and Porcelli F. 2005 Nonlinear saturation of tearing mode islands Phys. Rev. Lett. 95065001

[87] Wesson J.A. et al 1989 Disruptions in JET Nucl. Fusion 29641

[88] Buttery R.J., Sauter O., Akers R., Gryaznevich M., Martin R., Warrick C.D., Wilson H.R. and the MAST Team 2002 Neoclassical tearing physics in the spherical tokamak MAST Phys. Rev. Lett. 88125005

[89] Sauter O., Buttery R.J., Felton R., Hender T.C., Howell D.F. and Contributors to the EFDA-JET Workprogramme 2002 Marginal $\beta$-limit for neoclassical tearing modes in JET H-mode discharges Plasma Phys. Control. Fusion 441999

[90] Naylor G.A. 2010 An FPGA based control unit for synchronization of laser Thomson scattering measurements to plasma events on MAST Proc. 7th IAEA Technical Meeting on Control, Data Acquisition, and Remote Participation for Fusion Research (Aix-en-Provence, France, 15-19 June 2009) Fusion Eng. Des. 85 280-5 http://www.sciencedirect.com/science/article/pii/ S0920379610001833

[91] O’Gorman T., Naylor G., Gibson K.J., Huang B., McArdle G.J., Scannell R., Shibaev S., Snape J.A. and Thomas-Davies N. 2012 A field programmable gate array unit for the diagnosis and control of neoclassical tearing modes on MAST Rev. Sci. Instrum. 83 10E312

[92] Snape J.A., Gibson K.J., O’Gorman T., Barratt N.C., Imada K., Wilson H.R., Tallents G.J., Chapman I.T. and the MAST Team 2012 The influence of finite radial transport on the structure and evolution of $m / n=2 / 1$ neoclassical tearing modes on MAST Plasma Phys. Control. Fusion 54085001

[93] Thornton A.J., Gibson K.J., Chapman I.T., Harrison J.R., Kirk A., Lisgo S.W., Lehnen M., Martin R., Scannell R., Cullen A. and the MAST Team 2012 Plasma profile evolution during disruption mitigation via massive gas injection on MAST Nucl. Fusion 52063018

[94] Thornton A.J. et al 2013 Characterisation of disruption mitigation via massive gas injection on MAST Plasma Phys. Control. Fusion submitted

[95] Shevchenko V.F., De Bock M.F.M., Freethy S.J., Saveliev A.N. and Vann R.G.L. 2011 Two dimensional studies of electron Bernstein wave emission in MAST Fusion Sci. Technol. $59663-9$

[96] Shevchenko V.F., Vann R.G.L., Freethy S.J. and Huang B.K. 2012 Synthetic aperture microwave imaging with active probing for fusion plasma diagnostics J. Instrum. 7 P10016

[97] Urban J., Preinhaelter J., Diem S.J., Laqua H.P., Pavlo P., Shevchenko V., Taylor G., Vahala G., Vahala L. and Valovič M. 2009 EBW simulations in an experimental context J. Plasma Fusion Res. Ser. 8 1153-7 